\title{
Spin excitations in layered cuprates: a Fermi-liquid approach
}

\author{
I. Eremin ${ }^{1,2}$ and D. Manske ${ }^{3}$ \\ 1 Max-Planck Institut für Physik Komplexer Systeme, Dresden D-01187, Germany \\ E-mail: ieremin@mpipks-dresden.mpg.de \\ ${ }^{2}$ Institut für Mathematische/Theoretische Physik, \\ Technische Universität Carolina-Wilhelmina zu Braunschweig, Braunschweig D-38106, Germany \\ ${ }^{3}$ Institut für Theoretische Physik, ETH Zürich, Hönggerberg, CH-8093 Zürich, Switzerland \\ E-mail: manske@itp.ethz.ch
}

Received January 5, 2006

\begin{abstract}
We review some important aspects of the doping dependence of many physical properties of the high- $T_{C}$ cuprates based on a Fermi liquid-like approach. In particular, we show that the spin fluctuation-mechanism of superconductivity on the basis of a microscopic Eliashberg approach sup-

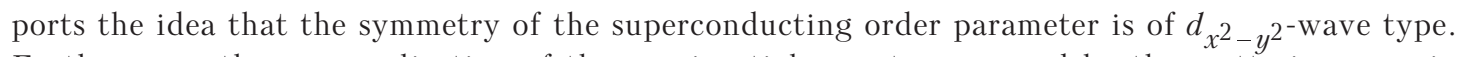
Furthermore, the renormalization of the quasiparticle spectrum caused by the scattering on spin fluctuations results in the so-called kink feature seen in ARPES. The peculiar momentum dependence of the spin fluctuations will result in a strong anisotropy of the renormalization at different parts of the first Brillouin zone and thus will lead to a strong anisotropy of the kink. Another important achievement of the microscopic Eliashberg approach is that the spin excitations spectrum renormalizes strongly below $T_{c}$ due to occurrence of superconductivity with a $d_{x^{2}-y^{2} \text {-wave order }}$ parameter which yields to a formation of the so-called resonance peak that can be viewed as a spin exciton. The topology of the Fermi surface and the momentum dependence of the superconducting gap explains the peculiar dispersion of the resonance peak in good agreement with experiments.
\end{abstract}

PACS: 74.72.-h, 74.20.Mn, 74.25.-q

Keywords: high- $T_{c}$ cuprates, Fermi-liquid approach, Brillouin zone.

\section{Introduction}

\subsection{Generic phase diagram}

In the last decade significant achievements in the understanding of the high- $T_{c}$ superconductivity phenomenon have been made on the basis of spin fluctuation-mediated Cooper pairing. Over the years a large amount of experimental data has been collected which suggest that an anisotropic superconducting order parameter is realized in the cuprate superconductors. Moreover, there are strong experimental indications that the superconducting order parameter is off $d$ wave type. A natural explanation of this symmetry originates from the concept of Cooper pairing due to exchange of antiferromagnetic spin-fluctuations [1]. This hypothesis is supported by the fact that the par- ent compounds of cuprate superconductors (like $\mathrm{La}_{2} \mathrm{CuO}_{4}$ or $\mathrm{YBa}_{2} \mathrm{Cu}_{3} \mathrm{O}_{6}$ ) are quasi two-dimensional antiferromagnetic insulators. Doping these systems with holes (for example, $\mathrm{La}_{2} \mathrm{CuO}_{4}$ with strontium or $\mathrm{YBa}_{2} \mathrm{Cu}_{3} \mathrm{O}_{6}$ with oxygen) results, first, in a transition into the metallic phase and, second, in the formation of unconventional superconductivity below a certain transition temperature $T_{C}$ that increases with increasing doping content towards its maximum value. Further doping of the system yields, however, in a decrease of $T_{C}$ and finally to the disappearance of superconductivity at some high doping level. Despite of the characteristic differences between the corresponding phase diagrams of various hole-doped cuprates, one can map all of them onto the so-called generic phase diagram. This is possible if one assumes 


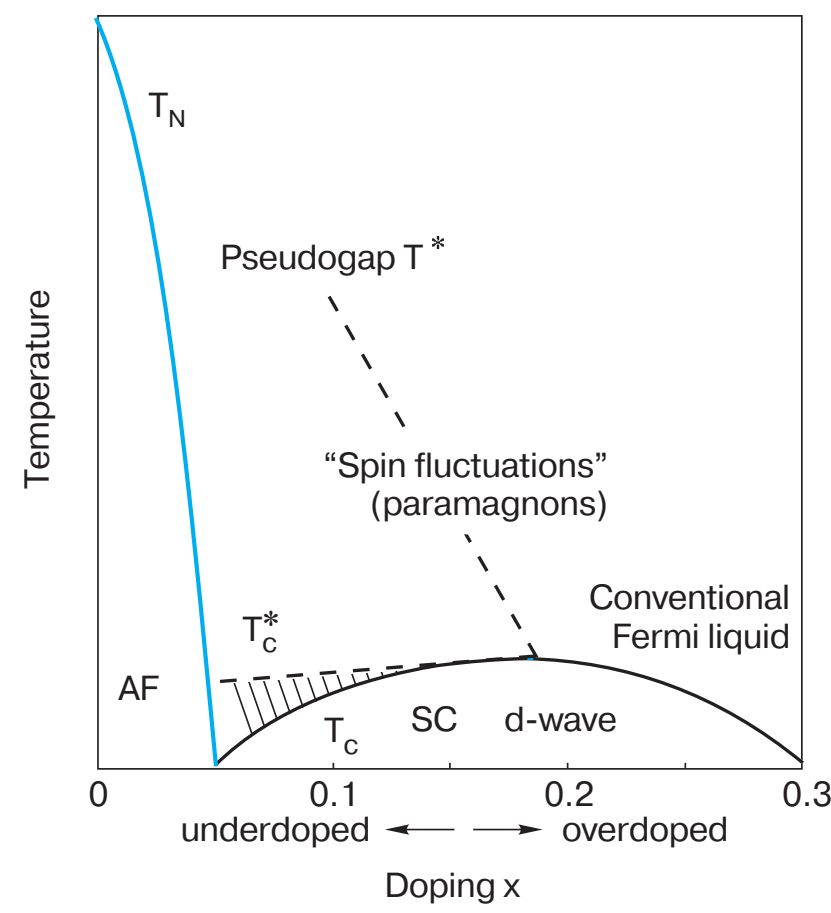

Fig. 1. Schematic phase diagram of hole-doped cuprates. High- $T_{C}$ superconductivity occurs in the vicinity of an antiferromagnetic phase transition. The corresponding superconducting order parameter below the superconducting transition temperature $T_{C}$ is of $d$-wave symmetry. In the overdoped region, i.e. $x>0.15$, the cuprates behave like a conventional Fermi liquid, whereas in the underdoped regime below the pseudogap temperature $T^{*}$ one finds strong antiferromagnetic (AF) correlations. As we will discuss below, Cooper pairing can mainly be described by the exchange of AF spin fluctuations (often called paramagnons). The doping region between $T_{C}$ and $T_{c}^{*}$ (shaded region) may be due to local phase-incoherent Cooper-pair formation. Only below $T_{C}$ these pairs become phase coherent.

that the processes relevant for the Cooper pairing and normal state dynamics already occur in a single $\mathrm{CuO}_{2}$ plane which is an important element of the crystal structure of the cuprates. In such a picture, the physical properties of each cuprate compound depend on the number of carriers within the $\mathrm{CuO}_{2}$ plane.

In Fig. 1 we show an illustration of the corresponding generic phase diagram for hole-doped cuprates that reveals the main features of the cuprates. One sees that superconductivity occurs in cuprates in a close vicinity to antiferromagnetism. Although antiferromagnetism and superconductivity seem not to coexist in cuprates, several experimental techniques [2-4] indicate the presence of short-range antiferromagnetic spin fluctuations even at optimal doping (i.e. maximum of $T_{c}$ ) and in overdoped samples. This suggests their possible importance for the Cooper pairing as well as for the anomalous normal state properties, in particular, close to antiferromagnetic transition. Certainly Landau's simple Fermi-liquid theory cannot be applied in the underdoped region. Moreover, there are two other curves on the phase diagram that do not refer necessarily to real phase transitions, but rather represent an anomalous crossover behavior of the cuprates in these regions. The first curve, $T^{*}(x)$, refers to the so-called pseudogap formation temperature. As indicated in many experiments [5], below $T^{*}$ the spectral weight at the Fermi level strongly decreases with decreasing temperature which is represented in gap-like features in the charge and spin responses. Another characteristic temperature, $T_{c}^{*}$, represents some experimental findings interpreted in terms of the so-called formation of (local) Cooper pairs without phase coherence [6]. There one further reports on a strong Nernst signal which is interepreted in terms of existing vortices [7]. Note, the behavior of the normal state spin and charge dynamics in the underdoped regime are far from being understood after twenty years of intensive research.

At the same time, on the other part of the phase diagram (so-called overdoped regime) the behavior of cuprates in the normal state can be well described within the concept of the Fermi liquid. This indicates that the system is characterized by a presence of the well-defined quasiparticles well above $T_{c}$, presence of a rigid band which width does not change significantly upon the changing of the dopant concentration. In such a situation the interaction between quasiparticles can be to a large extent described within perturbation theory. Moreover, the relative simplicity of the normal state seems to make an understanding of the superconducting properties of cuprates easier both theoretically and experimentally in the region of overdoped cuprates.

\subsection{Superconducting state}

Symmetry of the order parameter. In Fig. 1 we also show the $T_{c}(x)$ curve which has a characteristic shape $T_{c}(x) / T_{c}^{\max }=1-(x-0.16)^{2}$ as have been pointed out in Ref. 8. Below $T_{c}$ superconductivity occurs and it is believed to possess $d_{x^{2}-y^{2}}$-wave symmetry of the superconducting order parameter, i.e. $\Delta_{k}=$ $=\Delta_{0}\left(\cos k_{x}-\cos k_{y}\right) / 2$. The evidence for $d_{x^{2}-y^{2}}{ }^{-}$ wave pairing comes from several sources, and in particular from phase sensitive measurements [9]. Another remarkable feature about the superconducting state of high- $T_{c}$ cuprates is that they differ from conventional superconductors by having a small coherence length $\xi$. This length is usually associated with the average size of a Cooper pair, which is for conventional superconductors $\approx 500 \AA$. Therefore, the size of 
the Cooper pair is larger than the average distance between pairs resulting in a strong overlap of the corresponding wave functions. On the other hand, superconducting cuprates only have $\xi \approx 12-15 \AA$. Thus it is possible to find local pairs without long-range phase coherence. Another unusual feature relates to the ratio of $2 \Delta_{0} / k_{B} T_{c} \approx 5$ that is larger than the BCS ratio of 3.52. At the same time, such a ratio points towards a possible applicability of the strong-coupling Eliashberg-like theories for high- $T_{c}$ cuprates, at least in the overdoped and optimally-doped cuprates. However, the unconventional symmetry of the superconducting order parameter requires another mechanism than the exchange of phonons for the Cooper pairing.

Magnetic excitations. The idea of a spin-fluctuations mechanism of superconductivity conceptually is not new and has been originally proposed to describe the superfluid properties of ${ }^{3} \mathrm{He}$ [10]. In application to cuprates it has been first developed on the basis of a phenomenological approach which is based on the analysis of the experimental data $[11,12]$. In fact, the phenomenological approach has been proposed before the full development of the microscopic description of high- $T_{c}$ superconductivity, a description based on self-consistently solving the equations of the electron self-energy and the magnetic spin susceptibility in the frame of the Hubbard model was made. A detailed study of the temperature behavior of nuclear magnetic resonance (NMR) characteristics, such as the Knight shift and the spin-lattice relaxation time, and the inelastic neutron scattering (INS) intensity has shown that the detected anomalies can be explained by the presence of strong antiferromagnetic fluctuations near the antiferromagnetic wave vector $\mathbf{Q}=(\pi, \pi)$. Originally, Millis, Monien, and Pines [12] proposed a so-called Ornstein-Zernike form for the low-frequency magnetic susceptibility, $\chi(\mathbf{q}, \omega)$, in the normal state of hole-doped cuprates that allows for a sharp enhancement of fluctuations near Q:

$$
\chi(\mathbf{q}, \omega)=\frac{\chi_{\mathbf{Q}}}{1+\xi^{2}(\mathbf{q}-\mathbf{Q})^{2}-i \omega / \omega_{s f}} .
$$

Here, $\chi_{Q}$ is the value of the static spin susceptibility at the wave vector $\mathbf{Q}, \xi$ is the magnetic correlation length, and $\omega_{s f}$ is the characteristic frequency of spin fluctuations. For different materials these parameters have been found by fitting experimental data on NMR, since the general relationship relating the data to the dynamical spin susceptibility are well-known. The susceptibility determined in this manner was then used to calculate various quantities in the superconducting and normal phases of this compound, and the results of such calculations can be compared with the experimental data. In this way the main idea that antiferromagnetic spin fluctuations are important for the pairing process can be verified.

In the past, one of the biggest achievement of the spin-fluctuation mediated Cooper-pairing mechanism was a successful prediction of a correct symmetry of the superconducting order parameter in cuprates. In particular, in the phenomenological approach one first sets up an effective Hamiltonian. It must consist of two parts: $H=H_{0}+H_{\text {int }}$, where

$$
H_{0}=\sum_{\mathbf{k} \sigma} \varepsilon(\mathbf{k}) C_{\mathbf{k} \sigma}^{+} C_{\mathbf{k} \sigma}
$$

is the bare electron (hole) spectrum in the $\mathrm{Cu}-\mathrm{O}$ plane, and $H_{\text {int }}$ represents the interaction of electrons and spin fluctuations:

$$
H_{\text {int }}=\sum_{\mathbf{q}} g(\mathbf{q}) s(\mathbf{q}) S(-\mathbf{q}) .
$$

Here $s(\mathbf{q})$ is the electron spin operator,

$$
s(\mathbf{q})=\frac{1}{2} \sum_{\mathbf{k}} \sum_{\sigma \sigma^{\prime}} C_{\mathbf{k}+\mathbf{q} \sigma}^{+} \tau \sigma \sigma^{\prime} C_{\mathbf{k} \sigma^{\prime}},
$$

where $\tau$ represents the Pauli matrices, and $S(\mathbf{q})$ is the spin fluctuation operator, whose properties are determined by the spin-spin correlation function [the dynamical spin susceptibility tensor, defined by Eq. (1)]. The interaction is assumed to be of short-range type, with the result that $g(\mathbf{q})$ is weakly momentum dependent and may be set to a constant. In the phenomenological theory by Pines and coworkers, the coupling constant remains a free parameter of the theory and can be fixed by a comparison with experiment. In second order perturbation theory, the interaction of the electrons and spin fluctuations leads to the following effective electron-electron interaction shown in Fig. 5 and the corresponding expression for the self-energy of the one-particle Green's function in the normal state:

$\Sigma\left(\mathbf{k}, i \omega_{n}\right)=g^{2} T \sum_{m} \sum_{\mathbf{k}^{\prime}} \chi\left(\mathbf{k}-\mathbf{k}^{\prime}, i \omega_{n}-i \omega_{m}\right) G\left(\mathbf{k}^{\prime}, i \omega_{m}\right)$,

and superconducting state

$$
\begin{aligned}
& \Phi\left(\mathbf{k}, i \omega_{n}\right)=-g^{2} T \sum_{m} \sum_{\mathbf{k}^{\prime}} \chi\left(\mathbf{k}-\mathbf{k}^{\prime}, i \omega_{n}-i \omega_{m}\right) \times \\
& \times G\left(\mathbf{k}^{\prime}, i \omega_{m}\right) G\left(-\mathbf{k}^{\prime},-i \omega_{m}\right) \Phi\left(\mathbf{k}^{\prime}, i \omega_{m}\right),
\end{aligned}
$$

Eqs. (4) and (5) have to be combined with the Dyson equation,

$$
G\left(\mathbf{k}, i \omega_{n}\right)=\frac{1}{i \omega_{n}-\varepsilon(\mathbf{k})+\mu-\Sigma\left(\mathbf{k}, i \omega_{n}\right)},
$$

which produces a closed system of equations for finding the self-energy corrections for the normal and 
superconducting state. Here, $\chi\left(\mathbf{q}, i \omega_{l}\right)$ is the Matsubara Green's function of spin fluctuations which is related to the dynamic magnetic susceptibility by

$$
\chi\left(\mathbf{k}, i \omega_{l}\right)=-\int_{-\infty}^{+\infty} \frac{d \omega}{\pi} \frac{\operatorname{Im} \chi(\mathbf{q}, \omega)}{i \omega_{l}-\omega} .
$$

As one could see within this approach the dynamical spin susceptibility determines the properties of the normal and superconducting state. In order to discuss the symmetry of the superconducting order parameter it is enough to look on the weak-coupling limit, namely BCS gap equation which has the form at $T=0$ $\left(E_{\mathbf{k}}=\Delta_{\mathbf{k}}^{2}+\varepsilon_{\mathbf{k}}^{2}\right)$ :

$$
\Delta(\mathbf{k}, \omega)=-g^{2} \sum_{m} \sum_{\mathbf{k}^{\prime}} \frac{\chi\left(\mathbf{k}-\mathbf{k}^{\prime}, \omega\right)}{2 E_{\mathbf{k}^{\prime}}} \Delta\left(\mathbf{k}^{\prime}, \omega\right) .
$$

On of the immediate consequence of the Cooper pairing due to spin fluctuations is their repulsive nature in the singlet s-wave channel. Therefore, to overcome the minus in front of Eq. (8), the order parameter has to change sign. In high $-T_{c}$ cuprates the dynamical spin susceptibility is peaked at the antiferromagnetic wave vector, $\mathbf{Q}$, which connects the electronic states at the Fermi surface close the $( \pm \pi, 0),(0, \pm \pi)$ in the first Brillouin zone (BZ) (see Fig. 2 for the illustration).

Then, one could see that the natural solution of the gap equation for the particular topology of the Fermi surface and effective pairing interaction is the $d_{x^{2}-y^{2}}$-wave one. In particular, due to the change of

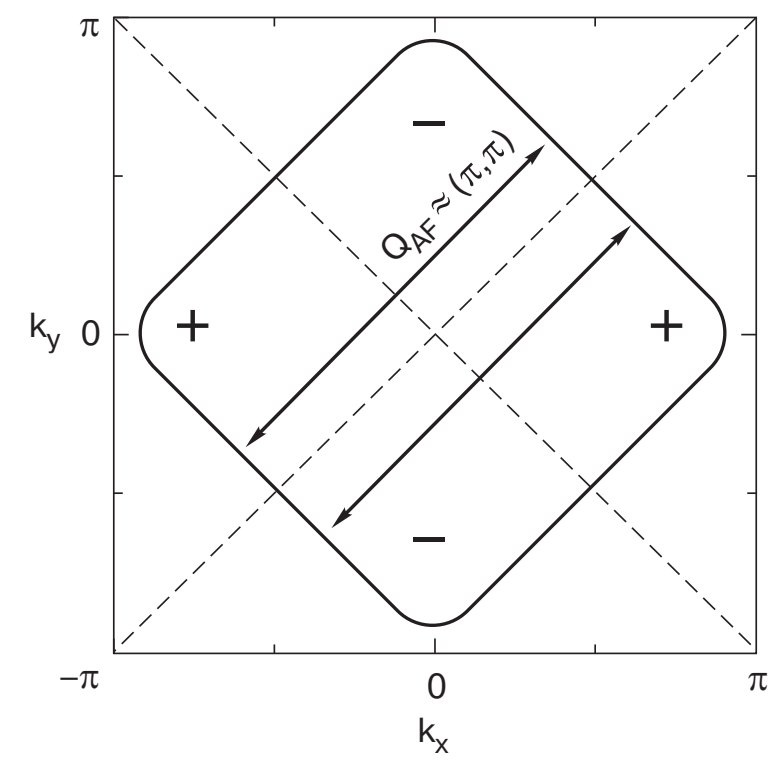

Fig. 2. Depicted Fermi surface for the layered cuprates in the first BZ. The arrows show the electronic states connected by the antiferromagnetic wave vector $\mathbf{Q}_{A F}$. The dotted lines and signs refer to the $d_{x^{2}-y^{2}}$-wave symmetry of the superconducting gap. sign of the order parameter the total minus in front of the gap equation is overcome and the solution of the gap equation is possible. Moreover, based on this phenomenological form of the spin susceptibility one can further determine $T_{c}$ and the phase diagram which to a large extent agrees with experiment. Note, such a solution does not exclude a possible sub-dominant contribution of the electron-phonon interaction if the latter is peaked at momenta $( \pm 0.4 \pi, 0)$, or $(0, \pm 0.4 \pi)$, for example. The sign of the order parameter for electronic states in the first $\mathrm{BZ}$ connected by those vectors still will be the same and thus, a further enhancement of $T_{c}$ is possible.

Despite the success of this phenomenological form of the spin susceptibility in describing the normal state spin dynamics it fails to explain the changes in the spin excitation spectrum in the superconducting state. In particular, in Fig. 3 we show the INS results for slightly underdoped $\mathrm{YBa}_{2} \mathrm{Cu}_{3} \mathrm{O}_{6.7}$ [13]. The intensity in INS is proportional to the magnetic structure factor that contains the imaginary part of the dynamical spin susceptibility. Thus, INS provides a direct probe of the spin susceptibility. As one sees, at the antiferromagnetic wave vector $\mathbf{Q}$ the normal state spin dynamics follows the Ornstein-Zernike behavior reflected by the rapid and linear increase of $\operatorname{Im} \chi(\mathbf{Q}, \omega)$ and a maximum at $\omega_{s f}$. However, the behavior of $\operatorname{Im} \chi(\mathbf{Q}, \omega)$ in the superconducting state is much different. The spin excitation spectrum strongly renormalizes and, first, becomes gapped at lower frequencies and then a sharp develops at $\omega_{\text {res }} \approx 33 \mathrm{meV}$ (it shifts to $41 \mathrm{meV}$ in optimally doped cuprates). The appearance of the resonance peak firstly reported in Ref. 14 suggests a strong renormalization of the spin fluctuation spectrum in the superconducting state that cannot be accounted by the simple phenomenological form of the spin susceptibility. In a recent experimen-

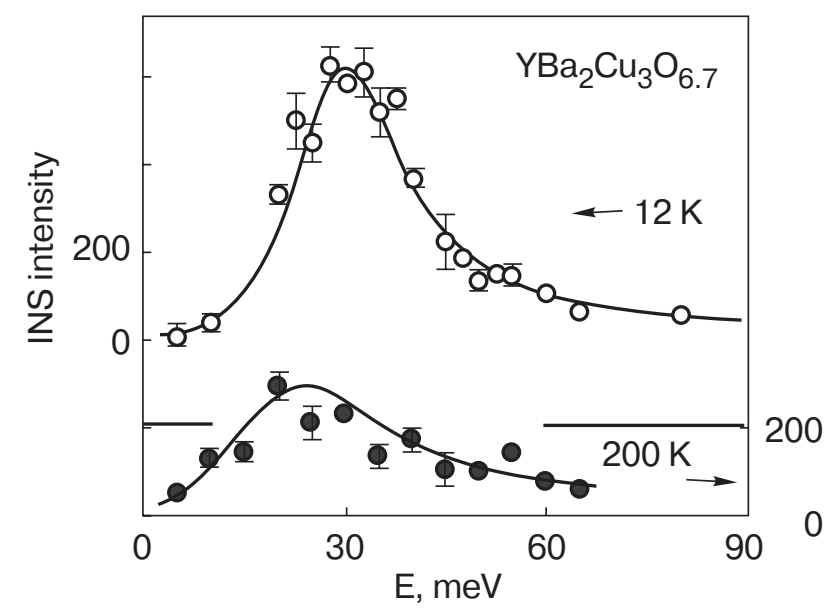

Fig. 3. INS results for optimally-doped $\mathrm{YBa}_{2} \mathrm{Cu}_{3} \mathrm{O}_{6.7}$ taken from Ref. [13] at the antiferromagnetic wave vector $\mathbf{Q}=(\pi, \pi)$ for the normal and superconducting state. 
tal work [15] an INS measurements have found also a resonant spin excitation in the overdoped and optimally-doped $\mathrm{Bi}_{2} \mathrm{Sr}_{2} \mathrm{CaCu}_{2} \mathrm{O}_{8+y}$ (BSCCO) with mode energies $E_{\text {res }}=38$ and $43 \mathrm{meV}$, respectively. This observation, together with the previous measurements in underdoped phase of $\mathrm{YBCO}$ indicates an approximately constant ratio $E_{\text {res }} / k_{B} T_{c}=5.4$ through the whole doping range. Very recently, the resonance peak emerged as a key factor in the phenomenology of the copper oxide superconductors and, in particular, of a single $\mathrm{CuO}_{2}$ plane due to the observation of the resonance like feature in the superconducting state of single $\mathrm{CuO}_{2}$ layer cuprate superconductor $\mathrm{Tl}_{2} \mathrm{Ba}_{2} \mathrm{CuO}_{6+x}$ near optimal doping [16].

One of the most appealing results concerning the resonant spin excitations is their dispersion away from $(\pi, \pi)$. In particular, as one moves away from $\mathbf{Q}$, the peak disperses downwards nearly quadratically and its intensity decreases rapidly, vanishing around $\mathbf{Q}_{0}=(0.8 \pi, 0.8 \pi)$. Recent INS experiments in the superconducting state of YBCO [17-19] have detected a new resonant magnetic excitation at incommensurate momenta, but at frequencies larger than $\omega_{\text {res }}(\mathbf{Q})$. Moreover, the high and low-energy excitations are separated from each by a region (so-called «silent bands») where no intensity has been observed. The whole dispersion of the resonant excitations is shown in Fig. 4.

Furthermore, recent experiments on the untwinned YBCO reveal a strong anisotropy of the resonant exci-

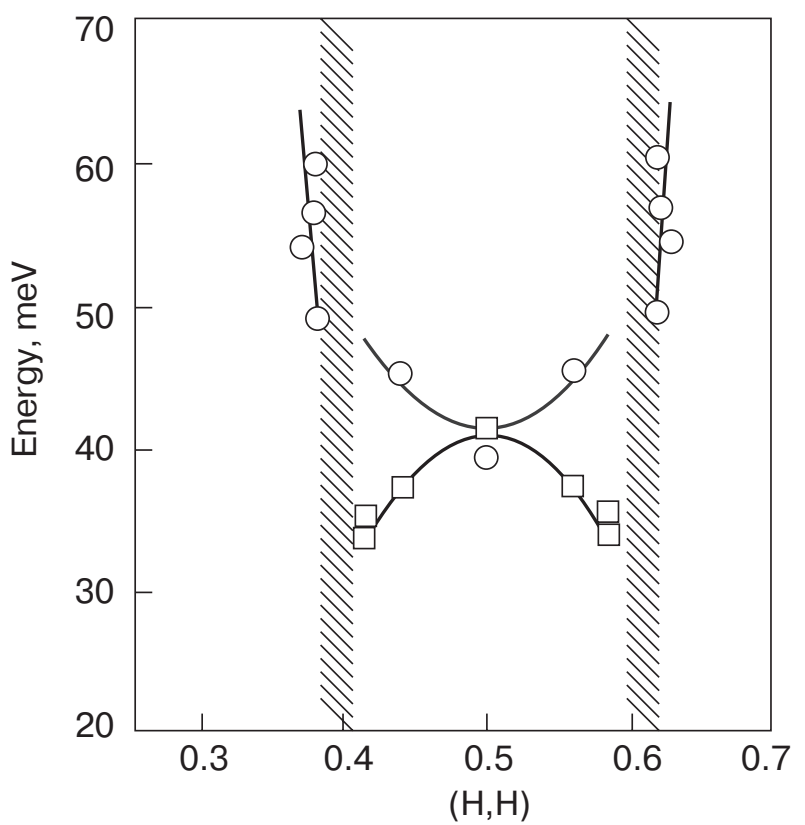

Fig. 4. Measured dispersion of the resonant excitations away from the antiferromagnetic wave vector $\mathbf{Q}_{A F}=0.5(\mathrm{H}, \mathrm{H})$ along the diagonal of the first BZ as taken from Ref. 17. The vertical dashed region indicates the position of the silent bands. tations below $\omega_{\text {res }}(\mathbf{Q})$. In particular, in the momentum space with a fixed energy, the resonant excitation form a so-called ring structure with four pronounced peaks along the bond directions. In the untwinned samples two of these peaks are suppressed, while two others remain [20]. All recent experimental facts are confirmed by various groups, and the observation in a different family of cuprate compounds indicate the necessity of the correct analysis of the spin fluctuations spectrum and its doping dependence in high- $T_{c}$ cuprates. This is a significant ingredient of a microscopic theory of cuprates must contain.

Elementary excitations. Another important question which one has to understand concerns the behavior of the elementary excitations in a $\mathrm{CuO}_{2}$ plane. Assuming the presence of strong antiferromagnetic spin fluctuations one expects, following Eqs. (4)-(7), a strong renormalization of the quasiparticle scattering rate and the corresponding energy dispersion. (The corresponding self-energy is illustrated in Fig. 5.) The effects of the scattering of the quasiparticles by the spin excitations can be seen by various experiments such as resistivity, optical conductivity, angle resolved photoemission (ARPES), tunneling and some others [21]. In particular, these days the development of ARPES experiment allows to get detailed information about the elementary excitations in the cuprates. This, for example, concerns a so-called «kink» feature, observed recently in various cuprate compounds [22-25]. Let us consider these experiments in more detail. Under well-defined assumptions it is believed that ARPES measures the spectral density of the electrons in the $\mathrm{CuO}_{2}$ planes:

$$
A(\mathbf{k}, \omega)=-\frac{1}{\pi} \frac{\operatorname{Im} \Sigma(\mathbf{k}, \omega)}{(\omega-\varepsilon(\mathbf{k})-\operatorname{Re} \Sigma(\mathbf{k}, \omega))^{2}+(\operatorname{Im} \Sigma(\mathbf{k}, \omega))^{2}},
$$

where $\varepsilon(\mathbf{k})=-2 t\left(\cos k_{x}+\cos k_{y}\right)+4 t^{\prime} \cos k_{x} \cos k_{y}-$ $-4 t^{\prime \prime}\left(\cos 2 k_{x}+\cos 2 k_{y}\right)-\mu$ is the unrenormalized tight-binding energy dispersion with hoping integrals between nearest, next-nearest, and so on sites. Due to

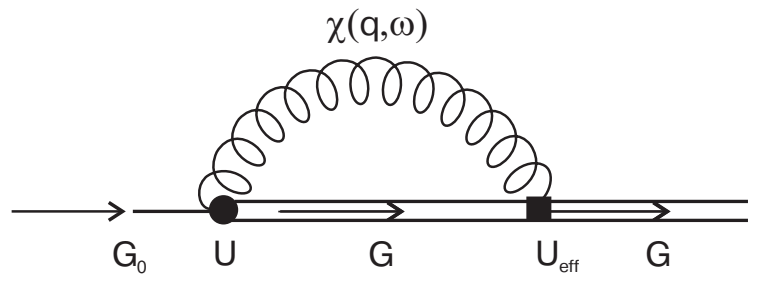

Fig. 5. Cooper-pairing in the cuprates due to coupling of carriers (holes or electrons) in $\mathrm{CuO}_{2}$ planes to antiferromagnetic spin fluctuations characterized by the spin-susceptibility $\chi(\mathbf{q}, 0)$ ( $G$ refers to matrix Green's function of quasi-particles). 
recent developments in ARPES, $A(\mathbf{k}, \omega)$ can be studied with high accuracy versus frequency at fixed momentum (so-called EDC) as well as function of momentum at fixed frequency (so-called MDC). One of the most important information that one gets by analyzing MDC and EDC is the renormalized energy dispersion $\omega_{k}$. In particular, the latter is obtained by studying the maximum of Eq. (9) which is satisfied if

$$
\omega-\varepsilon(\mathbf{k})-\operatorname{Re} \Sigma(\mathbf{k}, \omega)=0 .
$$

Note that MDC curves suit the purpose of defining of the energy dispersion much better due to a weaker momentum than frequency dependence of the self-en$\operatorname{ergy} \Sigma(\mathbf{k}, \omega)$.

In Fig. 6 we reproduce the results of Lanzara et al. [22] for the measured $\omega_{k}$ along $(0,0) \rightarrow(\pi, \pi)$ direction in the first Brillouine zone. One clearly sees that the curve shows change in its slope around $(50 \pm 15) \mathrm{meV}$. This is a so-called kink feature which was observed by several groups. As was argued in Ref. 26, the kink feature is seen along all directions in the $\mathrm{BZ}$ although it is doping and temperature dependencies are different. Originally, the kink feature was investigated along $(0,0) \rightarrow(\pi, \pi)$ direction. This is connected to the fact that along $(0,0) \rightarrow(\pi, 0)$ direction there are additional effects such as matrix elements and bilayer splitting which complicate the analysis of the experimental data. At the same, as we will argue, the main kink feature which could be related to

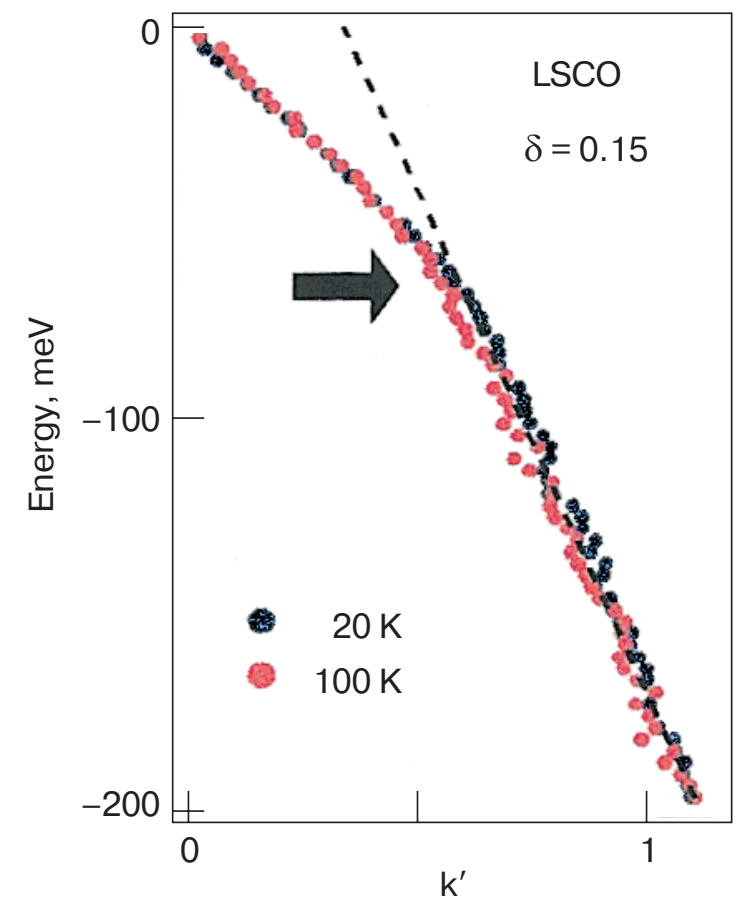

Fig. 6. ARPES results for the renormalized energy dispersion along $(0,0) \rightarrow(\pi, \pi)$ direction of the first Brillouine zone taken from Ref. 22. The horizontal axis shows the distance $\left(k-k_{F}\right)$ in the normalized units $k^{\prime}=k-k_{F}$. the feedback of superconductivity occurs along $(0, \pi) \rightarrow(\pi, \pi)$ due to the coupling to the resonant spin excitations. Originally the kink feature was attributed to a coupling of the itinerant carriers to phonons, in particular, to a longitudinal optical phonon mode at $60 \mathrm{meV}$ which was found to behave anomalously in several experiments [22]. On the other hand, the changes in the elementary excitations spectrum due to coupling of the carriers to spin fluctuations are also expected. Moreover, spin fluctuations would result in rather different frequency and momentum dependencies of the self-energy that will be reflected in the experiment than in phonon case. The latter seems to be confirmed by recent experimental results [26].

To conclude our Introduction, the intensive studies of high- $T_{C}$ cuprates in recent years have indicated that there is a strong interplay between strong electronic correlations and superconductivity in these materials which changes upon doping. In particular, a crossover from Fermi-liquid towards non-Fermi-liquid behavior occurs if one goes from the over- to underdoped regime and also an asymmetry with respect to hole or electron doping is found. Superconducting properties in cuprates reveal the $d$-wave superconducting order parameter and cannot be described in a frame of the standard BCS theory. Despite of the variety of theoretical and experimental problems that exist at the moment, in our review we will concentrate on the study of the spin excitations in the high- $T_{C}$ cuprates and their feedback on the elementary excitations. We will show that spin fluctuation-induced Cooper pairing allows to get a consistent and self-contained explanation of many experimental facts and to describe qualitatively the phase diagram of cuprates upon doping.

\section{Theory: generalized Eliashberg equations}

As we have mentioned in the Introduction the phenomenological approach based on the Cooper pairing via exchange of antiferromagnetic spin fluctuations explains some important facts on superconducting properties of cuprates. However, the complete picture requires a developing of a microscopic theory. A successful attempt in this direction was done independently by several groups [27-30] in the framework of a so-called FLEX approximation. Since the details of this method were already discussed in several reviews $[1,31,32]$ let us just briefly discuss the theoretical aspects of FLEX.

The simplest model that may describe the physics of a single $\mathrm{CuO}_{2}$ plane is a two-dimensional one-band Hubbard model which is an appropriate starting point of our calculations: 


$$
H=\sum_{\mathbf{k} \sigma} \varepsilon_{\mathbf{k}} c_{\mathbf{k} \sigma}^{+} c_{\mathbf{k} \sigma}+\frac{U}{2 N} \sum_{k k^{\prime} q \sigma} c_{\mathbf{k}, \sigma}^{+} c_{\mathbf{k}^{\prime},-\sigma}^{+} c_{\mathbf{k}^{\prime}+\mathbf{q},-\sigma} c_{\mathbf{k}-\mathbf{q}, \sigma} .
$$

Here, $c_{\mathbf{k} \sigma}^{+}\left(c_{\mathbf{k} \sigma}\right)$ are creation (annihilation) electron or hole operators on site i with spin $\sigma, U$ corresponds to the on-site effective Coulomb repulsion and $\varepsilon_{\mathbf{k}}$ is the tight-binding energy dispersion of the form

$$
\varepsilon_{k}=-2 t\left(\cos k_{x}+\cos k_{y}\right)+4 t^{\prime} \cos k_{x} \cos k_{y}-\mu,
$$

were $t$ and $t^{\prime}$ refers to the hopping of a hole(electron) between nearest, next-nearest sites on the square lattice and $\mu$ is the chemical potential that defines the doping. Here and in the following we set the lattice constant to unity. The description of the electronand hole-doped cuprates within a one-band approximation is possible if one takes into account different quasiparticle dispersion as observed in the experiment [33].

In the one-band model, the same electrons participate in the formation of antiferromagnetic fluctuations and in the Cooper pairing due to the exchange of such fluctuations. In this many-body problem both the magnetic susceptibility and the quasiparticle self- energy must be calculated self-consistently. We begin by writing the electron self-energy in the normal state, $\Sigma\left(\mathbf{k}, i \omega_{n}\right)$. In the Nambu representation, the $2 \times 2$ matrix $\Sigma\left(\mathbf{k}, i \omega_{n}\right)$ is given by the following expressions:

$$
\begin{aligned}
& \Sigma\left(\mathbf{k}, i \omega_{n}\right)=\sum_{\mathbf{k}^{\prime}, i \omega_{n}^{\prime}}\left[V_{s}\left(\mathbf{k}-\mathbf{k}^{\prime}, i v_{m}\right)-U^{2} \chi_{s 0}\left(\mathbf{k}-\mathbf{k}^{\prime}, i v_{m}\right)\right] \times \\
& \times \tau_{0} G\left(\mathbf{k}^{\prime}, i \omega_{n}^{\prime}\right) \tau_{0}+\sum_{\mathbf{k}^{\prime}, i \omega_{n}^{\prime}} V_{c}\left(\mathbf{k}-\mathbf{k}^{\prime}, i v_{m}\right) \tau_{1} G\left(\mathbf{k}^{\prime}, i \omega_{n}^{\prime}\right) \tau_{1} .
\end{aligned}
$$

Here, $\tau_{i}(i=0,1,2,3)$ are the Pauli matrices, $G\left(\mathbf{k}^{\prime}, i \omega_{n}^{\prime}\right)$ is an electron Greens function, and $i v_{n}=i \omega_{n}-i \omega_{n}^{\prime}$. The term $U^{2} \chi_{s 0}\left(\mathbf{k}-\mathbf{k}^{\prime}, i v_{m}\right)$ removes a double counting of diagrams in second order. $V_{s}$ and $V_{c}$ are the matrix elements of the electron-electron interaction due to spin and charge fluctuations:

$$
\begin{aligned}
& V_{s}\left(\mathbf{q}, i v_{m}\right)=\frac{3}{2} U^{2} \chi_{s}\left(\mathbf{q}, i v_{m}\right), \\
& V_{c}\left(\mathbf{q}, i v_{m}\right)=\frac{1}{2} U^{2} \chi_{c}\left(\mathbf{q}, i v_{m}\right),
\end{aligned}
$$

where $\chi_{c}\left(\mathbf{q}, i v_{n}\right)$ and $\chi_{s}\left(\mathbf{q}, i v_{n}\right)$ are the dynamical parts of the charge and spin susceptibility, respectively. In the random phase approximation (RPA) they are given by well-known diagrams corresponding to summation of loop diagrams:

$$
\begin{aligned}
& \chi_{s}\left(\mathbf{q}, i v_{m}\right)=\frac{\chi_{s 0}\left(\mathbf{q}, i v_{m}\right)}{1-U \chi_{s 0}\left(\mathbf{q}, i v_{m}\right)}, \\
& \chi_{c}\left(\mathbf{q}, i v_{m}\right)=\frac{\chi_{c 0}\left(\mathbf{q}, i v_{m}\right)}{1+U \chi_{c 0}\left(\mathbf{q}, i v_{m}\right)} .
\end{aligned}
$$

Here $\chi_{s 0}\left(\mathbf{q}, i v_{m}\right)$ and $\chi_{c 0}\left(\mathbf{q}, i v_{m}\right)$ are irreducible parts which in the single-loop approximation are given by the following formulas:

$$
\begin{gathered}
\chi_{s 0}\left(\mathbf{q}, i v_{m}\right)= \\
=-\frac{1}{2} T \sum_{k, i \omega_{n}} \operatorname{tr}\left[G\left(\mathbf{k}+\mathbf{q}, i \omega_{n}+i v_{m}\right) \tau_{0} G\left(\mathbf{k}, i \omega_{n}\right) \tau_{0}\right], \\
\chi_{c 0}\left(\mathbf{q}, i v_{m}\right)= \\
=-\frac{1}{2} T \sum_{k, i \omega_{n}} \operatorname{tr}\left[G\left(\mathbf{k}+\mathbf{q}, i \omega_{n}+i v_{m}\right) \tau_{3} G\left(\mathbf{k}, i \omega_{n}\right) \tau_{3}\right],
\end{gathered}
$$

where $\operatorname{tr}$ denotes the trace of a two-row matrix. The Green's function $G\left(\mathbf{k}, i \omega_{n}\right)$ and the self-energy $\Sigma\left(\mathbf{k}, i \omega_{n}\right)$ are connected by Dyson's equation:

$$
G\left(\mathbf{k}, i \omega_{n}\right)=\left[G_{0}^{-1}\left(\mathbf{k}, i \omega_{n}\right)-\Sigma\left(\mathbf{k}, i \omega_{n}\right)\right]^{-1},
$$

where in the case of singlet pairing the zeroth-order Green's function is given by

$$
G_{0}^{-1}\left(\mathbf{k}, i \omega_{n}\right)=i \omega_{n} \tau_{0}-\varepsilon_{\mathbf{k}} \tau_{3} .
$$

We expand the self-energy matrix in the standard form:

$$
\begin{aligned}
& \Sigma\left(\mathbf{k}, i \omega_{n}\right)=i \omega_{n}\left[1-Z\left(\mathbf{k}, i \omega_{n}\right)\right] \tau_{0}+ \\
& +\xi\left(\mathbf{k}, i \omega_{n}\right) \tau_{3}+\Phi\left(\mathbf{k}, i \omega_{n}\right) \tau_{1} .
\end{aligned}
$$

Here $Z$ is the mass renormalization factor that determines the renormalization frequency

$$
\tilde{\omega}\left(\mathbf{k}, i \omega_{n}\right)=\omega_{n} Z\left(\mathbf{k}, i \omega_{n}\right),
$$

$\xi\left(\mathbf{k}, i \omega_{n}\right)$ specifies the energy shift due to interaction of quasiparticles, and the function $\Phi\left(\mathbf{k}, i \omega_{n}\right)$ determines the superconducting gap:

$$
\Phi\left(\mathbf{k}, i \omega_{n}\right)=\frac{\Delta\left(\mathbf{k}, i \omega_{n}\right)}{Z\left(\mathbf{k}, i \omega_{n}\right)} .
$$

Equations (13) and (20) determine simultaneously three coupled nonlinear equations for these three quantities:

$$
\begin{aligned}
& \tilde{\omega}\left(\mathbf{k}, i \omega_{n}\right)=\omega_{n}+\sum_{\mathbf{k}^{\prime}, m}\left[V_{s}\left(\mathbf{k}-\mathbf{k}^{\prime}, i \omega_{n}-i \omega_{m}\right)+\right. \\
& \left.+V_{c}\left(\mathbf{k}-\mathbf{k}^{\prime}, i \omega_{n}-i \omega_{m}\right)\right] \frac{\tilde{\omega}\left(\mathbf{k}^{\prime}, i \omega_{m}\right)}{D\left(\mathbf{k}^{\prime}, i \omega_{m}\right)}
\end{aligned}
$$




$$
\begin{aligned}
& \xi\left(\mathbf{k}, i \omega_{n}\right)=-T \sum_{\mathbf{k}^{\prime}, m}\left[V_{s}\left(\mathbf{k}-\mathbf{k}^{\prime}, i \omega_{n}-i \omega_{m}\right)+\right. \\
& \left.+V_{c}\left(\mathbf{k}-\mathbf{k}^{\prime}, i \omega_{n}-i \omega_{m}\right)\right] \frac{\varepsilon_{\mathbf{k}^{\prime}}+\xi\left(\mathbf{k}^{\prime}, i \omega_{m}\right)}{D\left(\mathbf{k}^{\prime}, i \omega_{m}\right)}, \\
& \Phi\left(\mathbf{k}, i \omega_{n}\right)=-T \sum_{\mathbf{k}^{\prime}, m}\left[V_{s}\left(\mathbf{k}-\mathbf{k}^{\prime}, i \omega_{n}-i \omega_{m}\right)+\right. \\
& \left.+V_{c}\left(\mathbf{k}-\mathbf{k}^{\prime}, i \omega_{n}-i \omega_{m}\right)\right] \frac{\Phi\left(\mathbf{k}^{\prime}, i \omega_{m}\right)}{D\left(\mathbf{k}^{\prime}, i \omega_{m}\right)}
\end{aligned}
$$

where $D\left(\mathbf{k}^{\prime}, i \omega_{m}\right)$ is the denominator of the matrix electron Green's function:

$$
\begin{aligned}
& D\left(\mathbf{k}^{\prime}, i \omega_{m}\right)=\left[\tilde{\omega}\left(\mathbf{k}^{\prime}, i \omega_{m}\right)\right]^{2}+ \\
& +\left[\xi\left(\mathbf{k}^{\prime}, i \omega_{m}\right)\right]^{2}+\left[\Phi\left(\mathbf{k}^{\prime}, i \omega_{m}\right)\right]^{2} .
\end{aligned}
$$

These three equations must be augmented by an equation for the chemical potential

$$
n=\frac{1}{2}+2 T \sum_{n>0} \sum_{\mathbf{k}} \frac{\varepsilon_{k}+\xi\left(\mathbf{k}^{\prime}, i \omega_{m}\right)}{\Phi\left(\mathbf{k}^{\prime}, i \omega_{m}\right)}
$$

Equations (23)-(27) and (14)-(17) constitute a system of coupled equations for the electron Green's function and the dynamic susceptibilities of the superconductor and must be solved numerically for given values of the parameters $t, T, U$ and $n$ by iterations. For instance, we can plug the approximate values of the magnetic and dielectric susceptibilities into Eqs. (23)-(27) and calculate the values of $\tilde{\omega}\left(\mathbf{k}^{\prime}, i \omega_{m}\right), \xi\left(\mathbf{k}, i \omega_{n}\right)$, and $\Phi\left(\mathbf{k}, i \omega_{n}\right)$ which are functions that characterize the electron self-energy. Then, inserting these values into Eqs. (14)-(17), we calculate new values of the susceptibilities and plug them into Eqs. (23)-(27) being kernels of integral equations, which are then used to calculate new values of $\tilde{\omega}\left(\mathbf{k}^{\prime}, i \omega_{m}\right), \xi\left(\mathbf{k}, i \omega_{n}\right)$, and $\Phi\left(\mathbf{k}, i \omega_{n}\right)$. This procedure is repeated many times, as long as it is necessary to obtain a stable result. This scheme allows to calculate all properties of the system self-consistently such as the superconducting phase diagram, superconducting order parameter, energy dispersion and dynamical spin susceptibility which then have to be compared with experiment. An important remark which we would like to stress is that in contrast to the electron-phonon interaction, the spin-fluctuation-mediated pairing interaction is repulsive (in $\mathbf{k}$-space) and strongly momentum dependent. Therefore, as argued in connection with Eq. (8) the symmetry of the superconducting order parameter will be $d_{x^{2}-y^{2}}$-wave type. As already been mentioned the presented above theory can explain the symmetry of the superconducting gap and also main parts of the phase diagram of hole- and electron-doped cuprates. Thus, in the fol- lowing we will mainly analyze the behavior of the spin excitations and their feedback on the quasiparticle dispersion.

\section{Results and discussion}

\subsection{Spin excitations}

The investigation of the spin fluctuations spectrum is a key problem for understanding the physics of cuprates. Many researchers believe that the most significant problem which one has to clarify is the feedback of superconductivity on the spin excitation spectrum. This is of general interest, since in contrast to a phonon spectrum in conventional superconductors, spin excitations are strongly renormalized in the superconducting state that allows to identify «fingerprints» of the spin fluctuation scenario of superconductivity. For example, in many heavy fermions compounds and also recently discovered ferromagnetic superconductors the underlying pairing mechanism is not known, but very likely it is due spin fluctuations. Thus the knowledge about the fingerprints of a spin fluctuations scenario in cuprates is highly desirable.

Non-interacting part of the spin susceptibility. Let us first analyze the susceptibility of the non-interacting electron gas on a square lattice when we switch off the interaction between quasiparticles. To recall the behavior of the bare (non-interacting) susceptibility in the normal state we have to mention that in general on the square lattice there are eight characteristic scattering processes from $\mathbf{k}$ to $\mathbf{k}^{\prime}$ in the first BZ if a tight-binding band is used. The momenta $\mathbf{k}$ involved are those in which the boundary of the magnetic zone (defined by $\cos \mathbf{k}_{x}=-\cos \mathbf{k}_{y}$ ) crosses the Fermi surface (FS). Two of these scattering processes are direct

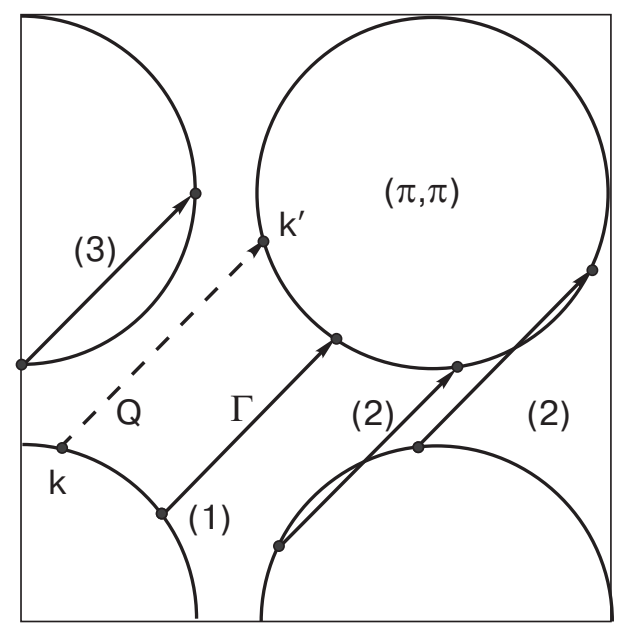

Fig. 7. Calculated Fermi surface for the for the first few BZ using a tight-binding energy dispersion from Eq. (12) with $t / t=0.4$ and optimal doping. The arrows indicate three different scattering processes as described in the text. 
involving momenta $\mathbf{k}-\mathbf{k}^{\prime}=\mathbf{Q}$, two involve umklapp scattering with $\mathbf{k}-\mathbf{k}^{\prime}=\mathbf{Q}-(2 \pi, 2 \pi)$ and four involve umklapp scattering with $\mathbf{k}-\mathbf{k}^{\prime}=\mathbf{Q}-(2 \pi, 0)$ and $\mathbf{k}-\mathbf{k}^{\prime}=\mathbf{Q}-(0,2 \pi)$. All these processes are degenerate at the antiferromagnetic wave vector and, thus, all eight processes equally contribute to the spin susceptibility yielding $\operatorname{Im} \chi_{0}(\mathbf{Q}, \omega) \approx-i \gamma \omega$ where $\gamma$ is the Landau damping rate. These transitions are illustrated in Fig. 7. In the superconducting state the susceptibility expression has a standard BCS form:

$$
\begin{aligned}
\chi_{0}(\mathbf{q}, \omega)= \\
=\sum_{\mathbf{k}}\left\{\frac{1}{2}\left[1+\frac{\varepsilon_{\mathbf{k}} \varepsilon_{\mathbf{k}+\mathbf{q}}+\Delta_{\mathbf{k}} \Delta_{\mathbf{k}+\mathbf{q}}}{E_{\mathbf{k}} E_{\mathbf{k}+\mathbf{q}}}\right] \frac{f\left(E_{\mathbf{k}+\mathbf{q}}\right)-f\left(E_{\mathbf{k}}\right)}{\omega-\left(E_{\mathbf{k}+\mathbf{q}}-E_{\mathbf{k}}\right)+i \delta}+\right. \\
+\frac{1}{4}\left[1-\frac{\varepsilon_{\mathbf{k}} \varepsilon_{\mathbf{k}+\mathbf{q}}+\Lambda_{\mathbf{k}} \Delta_{\mathbf{k}+\mathbf{q}}}{E_{\mathbf{k}} E_{\mathbf{k}+\mathbf{q}}}\right] \frac{1-f\left(E_{\mathbf{k}+\mathbf{q}}\right)-f\left(E_{\mathbf{k}}\right)}{\omega+\left(E_{\mathbf{k}+\mathbf{q}}-E_{\mathbf{k}}\right)+i \delta}+ \\
\left.+\frac{1}{4}\left[1-\frac{\varepsilon_{\mathbf{k}} \varepsilon_{\mathbf{k}+\mathbf{q}}+\Lambda_{\mathbf{k}} \Delta_{\mathbf{k}+\mathbf{q}}}{E_{\mathbf{k}} E_{\mathbf{k}+\mathbf{q}}}\right] \frac{f\left(E_{\mathbf{k}+\mathbf{q}}\right)+f\left(E_{\mathbf{k}}\right)}{\omega-\left(E_{\mathbf{k}+\mathbf{q}}-E_{\mathbf{k}}\right)+i \delta}\right\} .
\end{aligned}
$$

The imaginary part at $T=0$ and positive frequencies reads:

$$
\begin{gathered}
\operatorname{Im} \chi_{0}(\mathbf{q}, \omega)= \\
=\frac{1}{4} \sum_{\mathbf{k}}\left\{\left[1-\frac{\varepsilon_{\mathbf{k}} \varepsilon_{\mathbf{k}+\mathbf{q}}+\Delta_{\mathbf{k}} \Delta_{\mathbf{k}+\mathbf{q}}}{E_{\mathbf{k}} E_{\mathbf{k}+\mathbf{q}}}\right] \delta\left(\omega-E_{\mathbf{k}}-E_{\mathbf{k}+q}\right)\right\} .
\end{gathered}
$$

Here, $f\left(E_{\mathbf{k}}\right)$ denotes the Fermi function and $E_{\mathbf{k}}=\sqrt{\varepsilon_{\mathbf{k}}^{2}+\Delta_{\mathbf{k}}^{2}}$ is the dispersion of the Cooper pairs in the superconducting state. As one sees from Eq. (29), the imaginary part of the BCS spin susceptibility is zero below the characteristic frequency $\Omega_{c}(\mathbf{q})=\left|\Delta_{\mathbf{k}}\right|+\left|\Delta_{\mathbf{k}+\mathbf{q}}\right|$ which is the same for all eight scattering channels at $\mathbf{Q}$. Moreover, due to a $d_{x^{2}-y^{2}}$-wave symmetry of the superconducting gap one finds for the antiferromagnetic wave vector $\Delta_{\mathbf{k}+\mathbf{Q}}=-\Delta_{\mathbf{k}}$. As a result, the anomalous coherence factor gets enhanced and $\operatorname{Im} \chi_{0}(\mathbf{Q}, \omega)$ possesses a discontinuous jump at this frequency as shown in Fig. 8. The height of the jump depends on the coherence factor which is equal to 2 if perfect nesting $\left(\varepsilon_{k+Q}=-\varepsilon_{k}\right)$ would be present. Note this is in strong contrast to the conventional superconductors with $s$-wave symmetry of the superconducting gap. The latter will show a continuous enhancement of $\operatorname{Im} \chi_{0}(\mathbf{Q}, \omega)$ above the threshold frequency, but not a discontinuous jump. The jump in $d$-wave superconductors is important because simultaneously $\operatorname{Re} \chi_{0}(\mathbf{Q}, \omega)$ will display a logarithmic singularity at

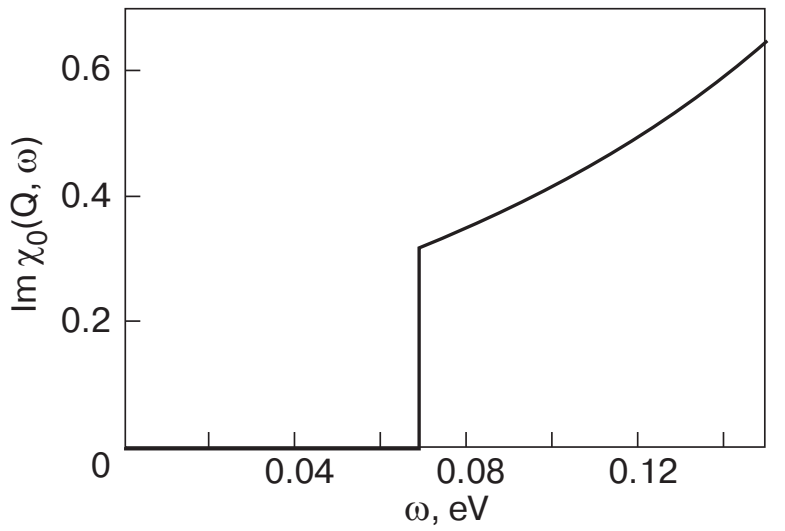

Fig. 8. Frequency dependence of $\operatorname{Im} \chi_{0}(\mathbf{Q}, 0)$ at the antiferromagnetic wave vector $\mathbf{q}=\mathbf{Q}$. It reveals a jump because of the $d$-wave symmetry of the superconducting gap.

the threshold frequency and, moreover, will be enhanced in comparison to the normal state behavior.

$R P A$ corrections. As a next step, within a conventional RPA, the imaginary part of the spin susceptibility can be written as:

$\operatorname{Im} \chi(\mathbf{Q}, \omega)=\frac{\operatorname{Im} \chi_{0}(\mathbf{Q}, \omega)}{\left(1-\operatorname{Re} \chi_{0}(\mathbf{Q}, \omega)\right)^{2}+U^{2}\left(\operatorname{Im} \chi_{0}(\mathbf{Q}, \omega)\right)^{2}}$.

One can immediately see that for any positive $U$, a resonance condition is fulfilled if $1=U \operatorname{Re} \chi_{0}(\mathbf{Q}, \omega)$, i.e. there is a pole in the RPA spin susceptibility which occurs for energies lower than the threshold. Therefore, a so-called spin exciton (or spin density collective mode) forms. With increasing $U$ the resonance shifts towards lower values of energy and increases its intensity as it is shown in Fig. 9.

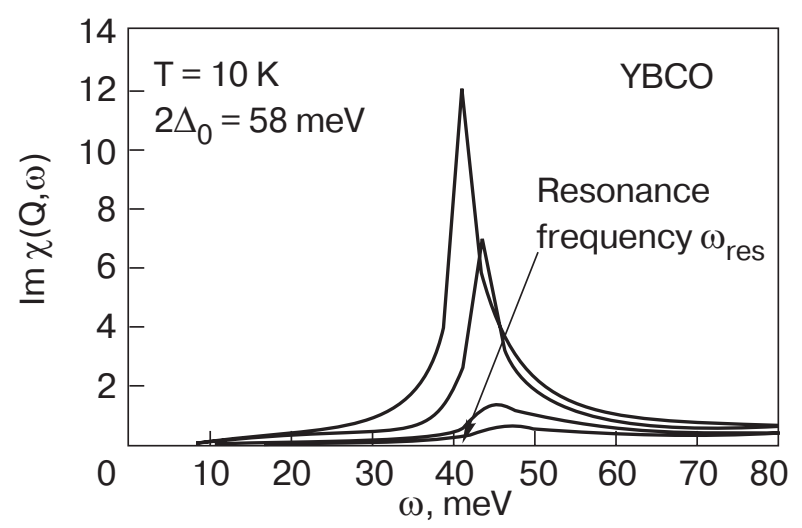

Fig. 9. Numerical results for the resonance peak in weak-coupling limit from Ref. 34 for optimal doping. Imaginary part of the RPA spin susceptibility (in units of states $/ \mathrm{eV})$ at wave vector $\mathbf{Q}=(\pi, \pi)$ for $U / t=1,2,3$, and 4 (from bottom to top). The resonance frequency was found at ${ }^{0}{ }_{\mathrm{res}}=41 \mathrm{meV}$. 


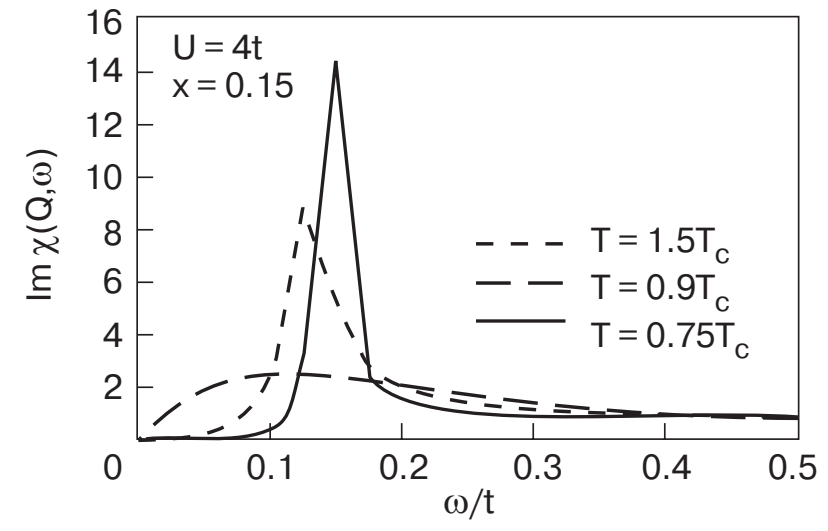

Fig. 10. Imaginary part of the RPA spin susceptibility at $\mathbf{Q}=(\pi, \pi)$ calculated within the FLEX approximation taken from Ref. 34 for optimal doping. For the normal state one gets $\omega_{s f}=0.1 t$ and in the superconducting state ${ }^{\omega^{\circ}}$ res $=0.15 t$. Assuming $t==250 \mathrm{meV}$ one finds that $\omega_{\mathrm{res}}=41 \mathrm{meV}$.

The self-consistency effects within the generalized Eliashberg formalism do not change the picture significantly, although deeper knowledge on the pairing interaction and its frequency dependence can be achieved. In Fig. 10 we demonstrate the results for Im $\chi_{R P A}(\mathbf{Q}, \omega)$ calculated within the FLEX approximation. In the superconducting state the resonance peak forms similar to the weak-coupling results. However, one has to remember here that the superconducting gap, $\Delta(\mathbf{k}, \omega)$, has been obtained in a self-consistent manner. For example one notices that the resonance peak evolves very fast below $T_{C}$ which agrees well with experimental data. The reason behind is that within Eliashberg theory the superconducting gap increase rapidly below $T_{c}$ and stabilizes the superconducting phase. Furthermore, a self-consistent scheme allows also to analyze the behavior of the resonance peak position with doping. In Fig. 11 we show the

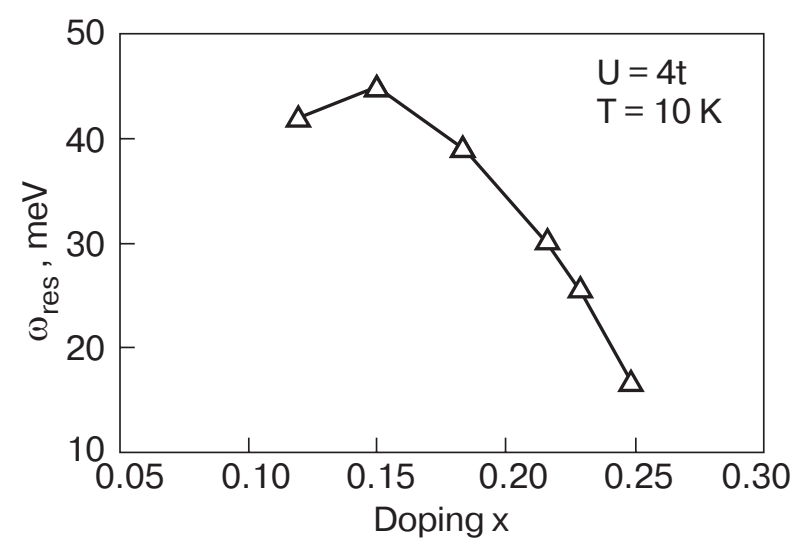

Fig. 11. Calculated results taken from Ref. 34 for the resonance frequency ${ }^{{ }}$res versus doping. In the overdoped regime one finds a constant ratio of ${ }^{0}$ res $/ T_{C}$. doping dependence of resonance peak position for various doping concentrations. One finds that $\omega_{\text {res }} \propto k_{B} T_{c}$ in the optimally and overdoped region in good agreement with experiment. Another interesting fact is a comparison with the normal state. Note, in the normal state the spin excitations spectrum within FLEX is characterized by a broad maximum at the energies of about $\omega_{s f}=0.1 t$ at the antiferromagnetic wave vector $\mathbf{Q}$. Therefore, the spin excitations are paramagnons, i.e. overdamped spin waves with a characteristic upward dispersion $\sim \mathbf{q}^{2}$. In the superconducting state one finds that the paramagnon feature disappears and the whole spectrum will be dominated by the dependence of the superconducting gap as a function of $\mathbf{q}$ on the Fermi surface. We will analyze this further by discussing the dispersion of the resonant excitations.

Dispersion of the resonance excitations. In the superconducting state away from $(\pi, \pi)$ the degeneracy in $\operatorname{Im} \chi_{0}\left(\mathbf{Q}_{i}, \omega\right)$ is lifted. In particular one has three critical frequencies, $\Omega_{c}^{(i)}(\mathbf{q})(i=1,2,3) . \Omega_{c}^{(1)}(\mathbf{q})$ is associated with direct process, $\Omega_{c}^{(2)}(\mathbf{q})$ with umklapp process $\mathbf{q}-(2 \pi, 0)$, and $\mathbf{q}-(0,2 \pi)$, and $\Omega_{c}^{(3)}(\mathbf{q})$ with an umklapp process $\mathbf{q}-(2 \pi, 2 \pi)$. For all of these processes the gap is still changing sign and, therefore, $\operatorname{Im} \chi_{0}\left(\mathbf{Q}_{i}, \omega\right)$ demonstrates three discontinuous jumps as illustrated in Fig. 12 and $\operatorname{Re} \chi_{0}\left(\mathbf{Q}_{i}, \omega\right)$ yields a logarithmic singularity.

However, $\operatorname{Im} \chi_{0}\left(\mathbf{Q}_{i}, \omega\right)$ is still zero only below the lowest jump, therefore the true resonance is only possible below the lowest jump which is in this case describe the direct scattering. Approaching $\mathbf{Q}_{0}=(0.8,0.8) \pi$ the jump due to the direct scattering approaches zero, because the states close the diagonal of the $\mathrm{BZ}$ are connected. The superconducting gap is zero there and, thus, $\operatorname{Im} \chi_{0}\left(\mathbf{Q}_{i}, \omega\right)$ is gapless there (see Fig. $12, c)$. Therefore, away from $(\pi, \pi)$ the reso-

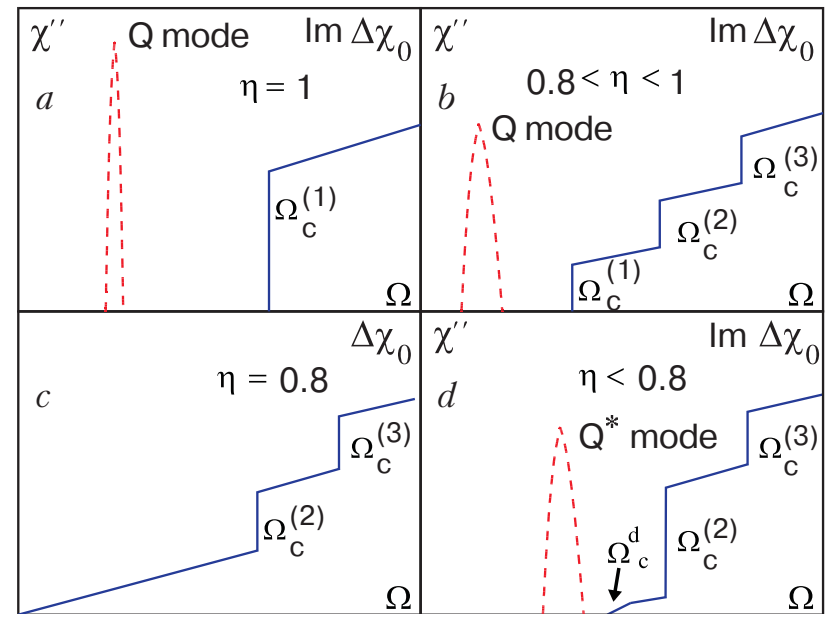

Fig. 12. Schematic behavior of $\operatorname{Im} \chi_{0}\left(\mathbf{Q}_{i}, \omega\right)$ and within RPA, Im $\chi\left(\mathbf{Q}_{i}, 0\right)$, for various momenta $\mathbf{q}=\eta(\pi, \pi)$. 
nance will disperse downwards following the momentum dependence of the superconducting gap at the Fermi surface. Due to the nodes of the superconducting gap, the downward dispersion should completely eliminate for the momenta close to $\mathbf{Q}_{0}=(0.8,0.8) \pi$ which explains the formation of the silent bands in the experiments. However, for the momenta smaller $\mathbf{q}<\mathbf{Q}_{0}$ the nodal points cannot be connected and a direct gap opens for the excitations into the $p-h$ continuum. This gap is not related to the superconducting gap and is determined by $\Omega_{c}^{d}(\mathbf{q}) \approx \mathbf{v}_{F}\left(\mathbf{q}-\mathbf{Q}_{0}\right)$. Due to the large Fermi velocity along the diagonal of the BZ the direct gap reaches fast the position of the second jump in $\operatorname{Im} \chi_{0}\left(\mathbf{Q}_{i}, \omega\right)$ which occurs due to umklapp processes. As a result the damping is removed and a true pole in the Eq. (32) is again possible, yielding a new resonance formation separated from the original resonance excitations by the silent band. This is clearly visible from Fig. 13 where we present our results for $\operatorname{Im} \chi\left(\mathbf{Q}_{i}, \omega\right)$ within RPA. It is perhaps interesting to note that away from $\mathbf{Q}_{0}$ the second «umklapp» resonance is again possible due to the fact that a coincidence of the both the direct gap and a second jump is required in order to see the resonance. Furthermore, both modes are well separated in energy and momentum. We would like to stress that within a spin exciton and Fermi liquid-like scenario for the resonance peak for a given momentum only one single real pole is possible. Moreover, the intensity of the umklapp resonance is about 5 times smaller that the original resonance at $(\pi, \pi)$ which is due to the fact that the new resonance excitations lie closer to the continuum than the original resonance at wavevector Q. Furthermore, the dispersion of the umklapp resonance is nearly vertical and disappers upon reaching the continuum. The origin of such a steep dispersion is

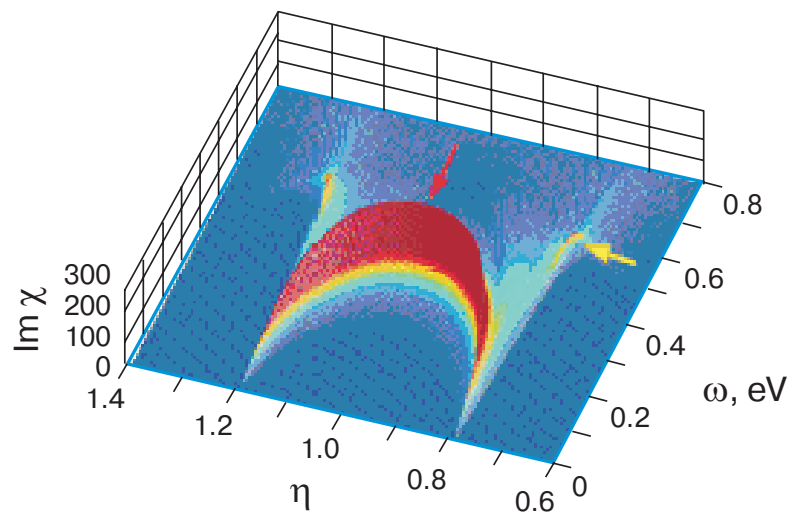

Fig. 13. RPA results for magnetic excitations in a

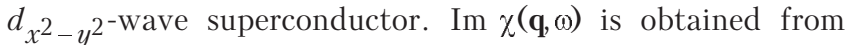
Eq. (36) as a function of momentum (along the diagonal $\mathbf{q}=\eta(\pi, \pi))$ and frequency in the superconducting state taken from Ref. 35. The arrows indicate the position of the resonance modes.

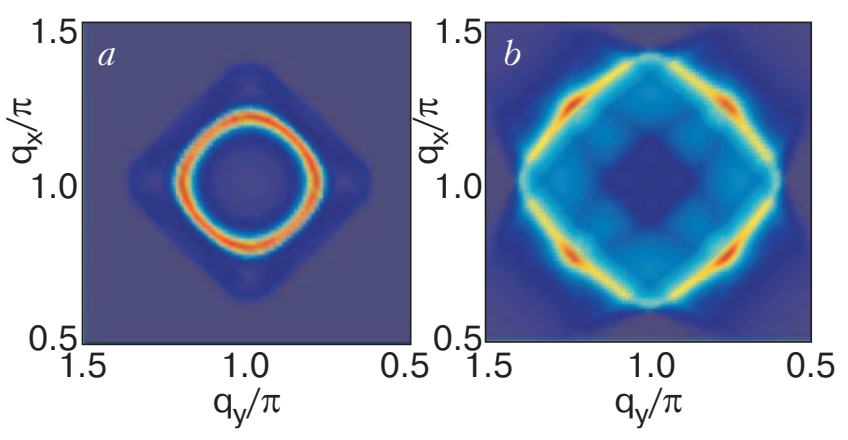

Fig. 14. Intensity patterns of the resonance excitations for $\omega=30 \mathrm{meV}(a)$ and $\omega=57 \mathrm{meV}(b)$ taken from Ref. 35.

governed by the Fermi velocity along the nodal direction.

Another interesting fact which further separates both resonance excitations is their intensity patterns in the momentum space for fixed frequencies is the following: In Fig. 14 we show the intensity pattern for the $\omega=30 \mathrm{meV}\left(\omega<\omega_{\text {res }}(\mathbf{Q})\right)$ and $\omega=57 \mathrm{meV}$ $\left(\omega>\omega_{\text {res }}(\mathbf{Q})\right)$. While for the original resonant excitations (so-called Q-mode in notation of Ref. 35) the maxima are certainly along the bond directions, for the novel mode (so-called $Q^{*}$-excitations) are along the diagonal of the BZ. The rotation of the maxima reflects the qualitative difference in the origin of both modes. In the first case the electrons which scatter along the bonds are located further away from the nodes than those which scatter along the diagonal. Thus, the intensity of the resonance is stronger for the bond direction. On the contrast, the resonance arises due to a rapid opening of the direct gap in the $p-h$ continuum and its coincidence with the discontinuous jump due to umklapp scattering. The latter is only possible along the diagonal of the BZ. In Ref. 35 it also has been found that the intensity pattern for the «upward» dispersion of the resonance remains always along the diagonal direction. However, the situation may change if for some reason the structure of the $p-h$ continuum modifies in a certain way. In particular, it may happen that for some specific band structure parameters the border of the $p-h$ continuum along the bonds lies higher than the diagonal direction. Then, the resonance excitations for the $Q{ }^{*}$-mode will also be along the bond directions. However, this occurs only very close to the border of $p-h$ continuum.

Influence of orthorhombic distortions. One of the interesting question is: what will happen to the resonant excitations in the presence of orthorhombic distortions. This is, in particular, intriguing due to the recent INS experiments on untwined YBCO [20]. Within a Fermi-liquid description the influence of the orthorhombic distortion can be taken into account via 
the modification of the tight-binding parameters of the model and an additional $s$-wave contribution to the superconducting gap. Most importantly, for the hopping along the bond directions one has to take $t_{x} \neq t_{y}$. In YBCO one finds due to the presence of the underlying $\mathrm{CuO}$-chains that the lattice constant $b>a$. Naively, one would expect that the hopping matrix element along the chains should be smaller than perpendicular to them. This will be the case for the pure two-dimensional model. However, in reality the plane states are hybridized with the chain states. The latter are metallic and thus, enhance the conductivity along the chain direction in the $\mathrm{CuO}_{2}$ plane. This assumption is further supported by experimental data [36]. Therefore, in order to model correctly the tight-binding parameters in the two-dimensional model Hamiltonian one has to assume $t_{a}<t_{b}$. This will produce the main effect in the calculations. Since the resonance excitations are sensitive to the topology of the Fermi surface, its change will affect the intensity of the peaks along the bond direction as shown in Fig. 15. In particular, due to the change of the hopping parameter, the Fermi surface around $( \pm \pi, 0)$ points will shift away and $(0, \pm \pi)$ shrinks towards these points, leading in the extreme case even towards closing of the Fermi surface around these points. However, this necessarily should not take place. The important results, however, is that due to the change of the topology of the Fermi surface, the phase space along $\mathrm{x}$ and along the $\mathrm{y}$-direction is changing. In addition, the superconducting gap will not be pure $d$-wave and an inclusion of the small admixture of the s-wave component is required.

In Fig. 16 we show the results for the RPA spin susceptibility as a function of the momentum for a constant energy of $35 \mathrm{meV}$. On can see that inclusion of

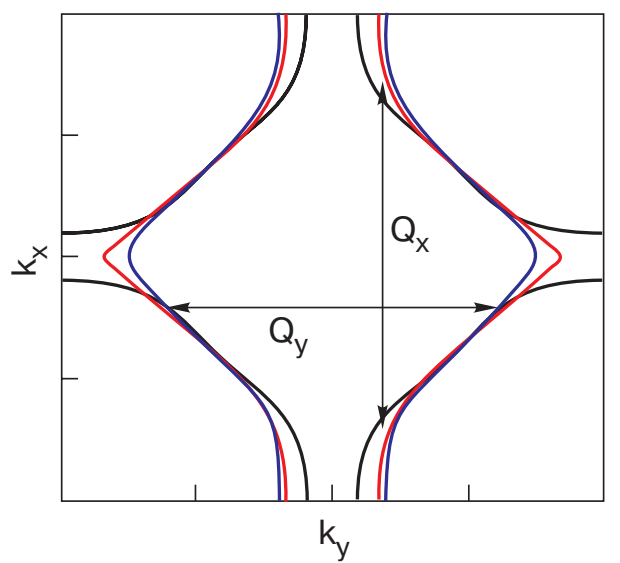

Fig. 15. Illustration of the possible drastic changes of the Fermi surface due to various parameters of the orthorhombicity, $\delta_{0}\left(t_{a}=t\left(1-\delta_{0}\right), t_{b}=t\left(1+\delta_{0}\right)\right)$ as adopted from Ref. 37. The arrows show the change of the phase space for the bond scattering.

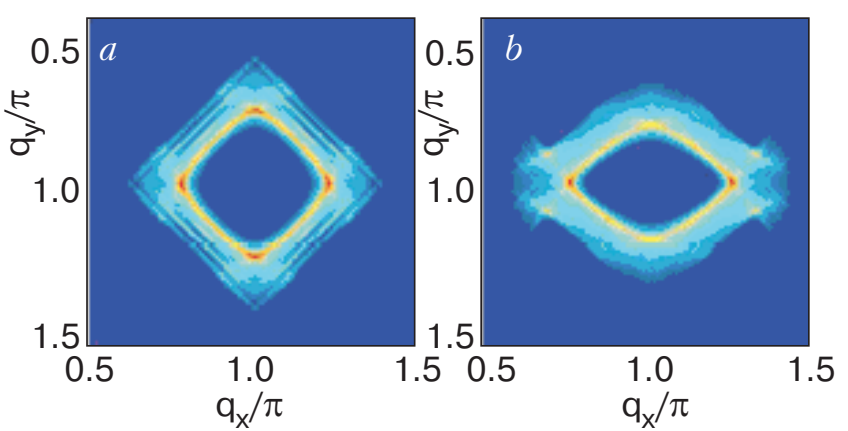

Fig. 16. Calculated normalized two-dimensional intensity plot of $\operatorname{Im} \chi_{R P A}(\mathbf{q}, 0)$ for constant energy of $35 \mathrm{meV}$ taken from Ref. 37, (a) without, (b) with inclusion of orthorhombicity $\left(\delta_{0}=-0.03\right)$. Note that two of the four incommensurate peaks are suppressed.

the orthorhombic distortions results in the suppression of two peaks along $y$-axis. This is a direct consequence of the reduction of the phase space illustrated in Fig. 15. Note this agrees well with the available experimental data [20].

Comparison with experiment. Finally we would like to note that surprisingly a simple Fermi liquid approach works rather well in explaining many features of the spin excitations in the superconducting state like the formation of the resonance peak at the antiferromagnetic wave vector $\mathbf{Q}=(\pi, \pi)$ and its peculiar dispersion including silent bands observed away from $(\pi, \pi)$. At the same time one should note certain difficulties. In particular, within the spin exciton scenario of the resonance peak there is always only one single pole at a given momenta as a function of frequency which yields a resonance peak formation. Therefore, the resonance dispersion does not form a so-called $X$-shape which is often claimed to be observed in the experiment [17]. This question has to be further clarified experimentally and theoretically. Another problem concerns the behavior of the resonant excitations in the pseudogap region of underdoped cuprates. There the spin excitations develop well above $T_{c}$ and exhibit slight renormalization in the superconducting state. Moreover, there is a surprising similarity of the spin excitations in the superconducting cuprates [17-19] and their non-superconducting «striped» counterpart [38] indicating a certain cross-over between the two systems. These aspects cannot be covered within a Fermi-liquid type theory developed here and is a subject of future studies.

\subsection{Elementary excitations}

For understanding the high- $T_{c}$ cuprates their low-energy excitations are of central significance. Thus we start the discussion of theoretical results by 
analyzing the spectral density in the normal state and compare it with available experimental data on ARPES that study in details the spectral density and in particular the renormalized energy dispersion. One general grounds one expects that antiferromagnetic spin fluctuations exist relatively close to the Fermi energy. Therefore, if one goes below the Fermi level the quasiparticles below some energy will not feel the effect of the scattering and will behave more like in a conventional Fermi-liquid picture. A similar effect is expected for electron-phonon interaction, but the behavior of the self-energy in the case phonons and spin fluctuations qualitatively differ from each other. This may help to see that the main effects arises from spin fluctuations.

In Fig. 17 we show results of Ref. 39 for the frequency dependence of the spectral density for different momentum along $(0,0) \rightarrow(\pi, 0)$ and $(0,0) \rightarrow(\pi, \pi)$ directions using the parameters for hole-doped cuprates and calculated as described in the previous section. The curves in Fig. 17 are analogous of a so-called EDC curves measured in the experiment. One clearly sees that both the width of the curves (which is proportional to $\operatorname{Im} \Sigma(\mathbf{k}, \omega)$ ) and position of the maximum (that represents a renormalized dispersion) are changing approximately at $(65 \pm 15) \mathrm{meV}$. Moreover, one could also notice that the changes along $(0,0) \rightarrow(\pi, 0)$ are more pronounced reflecting stronger nesting of the Fermi surface in this direction. Remarkably this is also the case in the experiment as one sees on Fig. 6. In order to see the «kink» structure in the dispersion we show in Fig. 18 the positions of the peaks shown in Fig. 17 as a function of $\left(\mathbf{k}-\mathbf{k}_{f}\right)$ for different temperatures above and below $T_{c}$. The

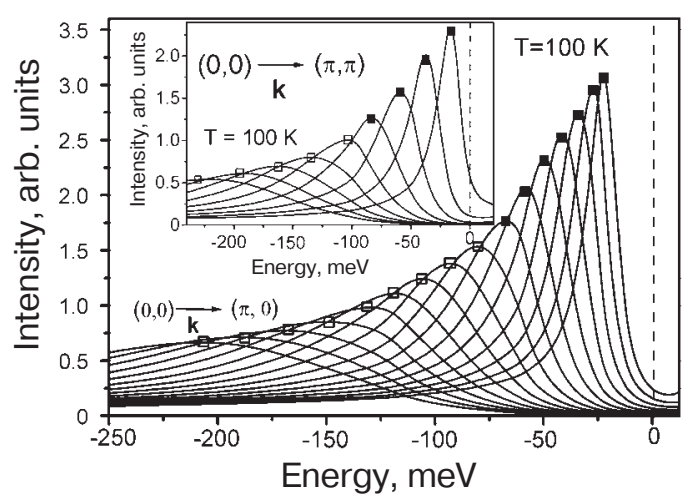

Fig. 17. Calculated spectral density from Ref. 39 of the quasiparticles in hole-doped cuprates at $T=100 \mathrm{~K}$ along $(0,0) \rightarrow(\pi, 0)$ and $(0,0) \rightarrow(\pi, \pi)$ (inset) directions of the first $\mathrm{BZ}$. The dashed line denotes the unrenormalized chemical potential. In both directions at energies approximately (65 \pm 15 ) $\mathrm{meV}$ a kink occurs since the velocity of quasiparticles changes.

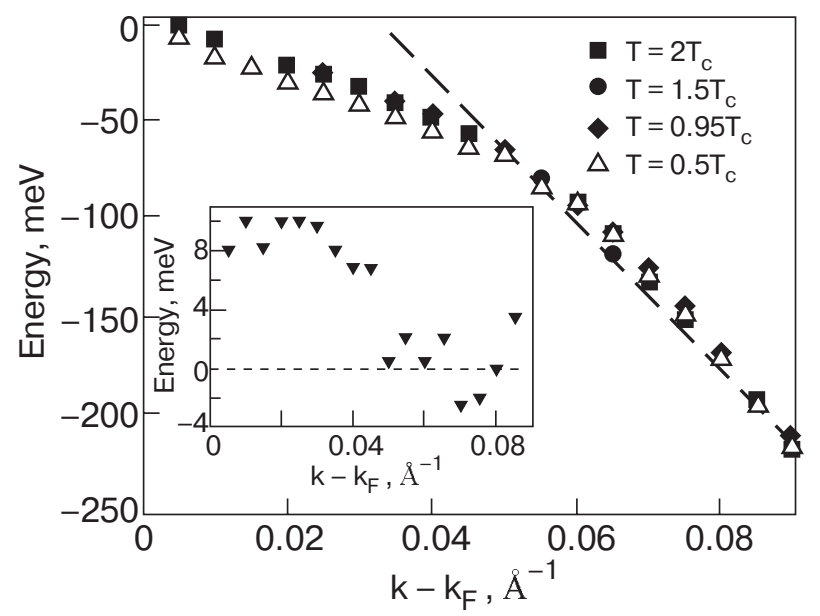

Fig. 18. Positions of the peaks in the spectral density $A(\mathbf{k}, 0)$ versus $\left(\mathbf{k}-\mathbf{k}_{f}\right)$ (energy dispersion) along the $(0,0) \rightarrow(\pi, 0)$ direction of the BZ calculated in Ref. 39 for optimally hole-doped cuprates. Inset: change in the peak positions of $A(\mathbf{k}, 0)$ in the superconducting state. Note, in underdoped cuprates the kink feature shifts to lower frequencies due to decreasing of ${ }^{0} s f$. In the overdoped regime the kink feature should become less pronounced and isotropic in different direction of the BZ.

slope of all curves changes at $(65 \pm 15) \mathrm{meV}$ reflecting the changes in the self-energy and, most importantly, weakly depend on temperature. These results are also in good agreement with experimental data, however, note that experimentally the kink feature is more often and easier extracted from the MDC curves.

However, this difference relates mainly just to the technical aspects in the experiment, since it is much easier to analyze the intensity of MDC Lorentzian curves than the EDC ones, in both cases the resulting dispersion should not differ. In the superconducting state only small changes in the dispersion are obtained (see inset) which is also in agreement with experiment [41]. It is remarkable that for electron-doped superconductors with a different dispersion we get no kink feature up to frequencies about $100 \mathrm{meV}$. The reason behind this is that spin fluctuations spectrum in electron-doped cuprates has no pronounced structures at low frequencies and fewer density of states.

One of the main experimental results is that the kink feature is more pronounced along $(0,0) \rightarrow(\pi, 0)$ direction, i.e. the change of the slope in the dispersion, is larger than along $(0,0) \rightarrow(\pi, \pi)$ direction $[25,26]$. However, the position is almost the same in all direction of the Brillouine zone. In the case of scattering of quasiparticles on antiferromagnetic spin fluctuations one expects this behavior due to the pronounced nesting properties of the Fermi surface around $( \pm \pi, 0)$ points of the Brillouine zone. Thus, the 

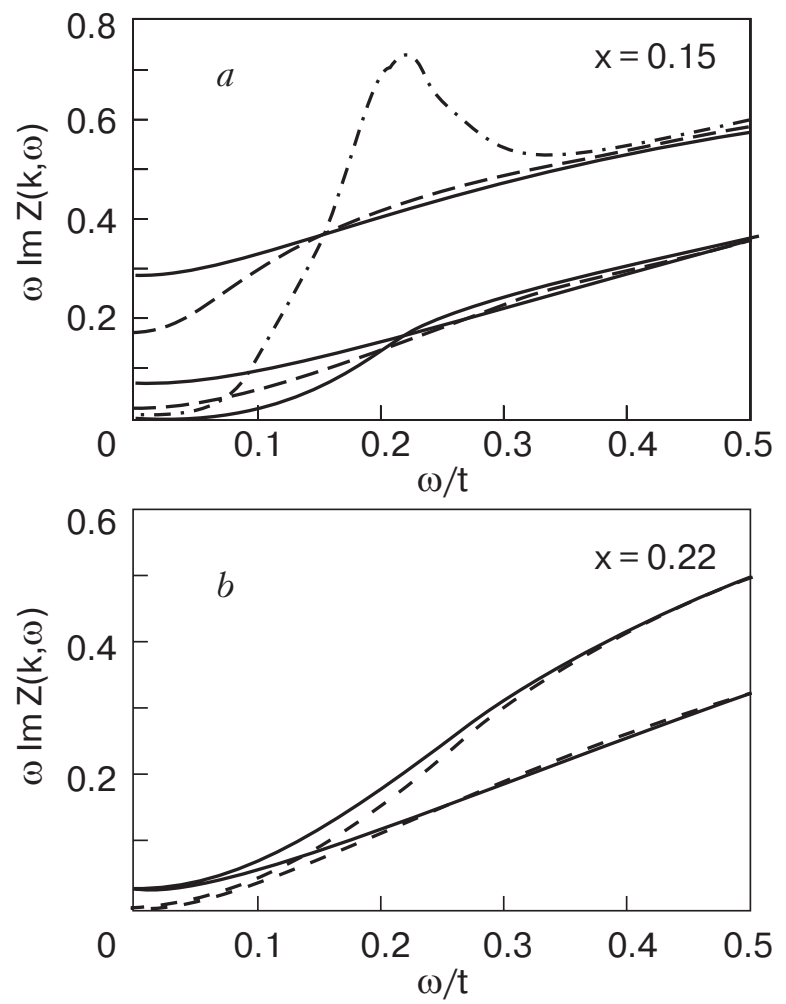

Fig. 19. Comparison of the quasiparticle damping $\Gamma=\omega \operatorname{Im} Z(\mathbf{k}, \omega)$ in the normal state for $(a)$ optimal doping $(x=0.15)$ and for $(b)$ the overdoped case $(x=0.22)$ taken from Ref. 40. The solid curves are calculated at $T=2 T_{C}$ and the dashed curves refer to $T=1.05 T_{c}$. The upper curves correspond to the antinodal wavevector $\mathbf{k}_{a}=(0.15 \pi, \pi)$ whereas the lower curves correspond to the nodal one $\mathbf{k}_{n}=(0.41 \pi, 0.41 \pi)$ both on the Fermi line. For a comparison, we show in $(a)$ also results for the superconducting state ( $T=0.75 T_{C}$, dashed-dotted curve). Note that for both doping concentrations a linear behavior for larger frequencies and a vanishing anisotropy for $\omega \rightarrow 0$ in the overdoped case.

changes of the self-energy and correspondingly in the quasiparticle damping will be stronger there. In order to see this in more details we show in Fig. 19 the evolution of the $\omega \operatorname{Im} Z(\mathbf{k}, \omega)$ versus frequency for different doping and temperatures at different points of the $\mathrm{BZ}$ calculated within the FLEX approximation in the normal and superconducting state. Note the strong anisotropy for $\omega \rightarrow 0$ for optimal doping between the nodal point $(0.41 \pi, 0.41 \pi)$ and the so-called antinodal point $(0.15 \pi, \pi)$ of the BZ. In particular, in the normal state the behavior of the quasiparticle damping is linear at the antinodal point and more quadratic Fermi-liquid like at the nodal direction. This further enhances with decreasing temperature. Therefore, the kink should be more pronounced along $(0,0) \rightarrow(\pi, 0)$ direction. Moreover, the important change happens in the superconducting state. While along the nodal direction the effect of superconductivity is relatively weak, the change at the antinodal point is more pronounced. Therefore, an additional structure is expected in the dispersion along $(0,0) \rightarrow(\pi, 0)$ direction of the BZ which was found recently [25]. This further confirms that the kink results from spin fluctuations. Other important changes occurs if one goes towards overdoped compounds as we show in Fig. 19, $b$. In this case the anisotropy between nodal and anti-nodal direction almost disappears. Therefore, the kink feature will be the same for the overdoped regime. This can be further studied in ARPES experiments. In contrast to the overdoped case, in the underdoped regime the anisotropy of the kink should become more visible and also the Fermi velocity should change along the Fermi line. Moreover, due close vicinity to an antiferromagnetism one expects the shift of the kink position towards lower values as was observed in the experiment $[25,26]$.

As we have already mentioned, a very naive explanation for the kink feature is that the quasiparticles below the Fermi level will not experience the scattering on spin fluctuations. In other words the antiferromagnetic wave vector $\mathbf{Q}_{\text {pair }}$ will not connect these holes on different Fermi arcs with each other. Then, these holes will behave in according to the standard Fermi liquid theory. There is a wide discussion whether or not layered cuprate superconductors behave like conventional Fermi liquids. Earlier experiments reveal non-Fermi liquid properties, in particular a linear resistivity $\rho(T)$ for optimal doping, non well-defined quasiparticle peaks above the superconducting transition temperature $T_{c}$, and a strong temperature dependence of the uniform spin susceptibility observed by nuclear magnetic resonance [42]. Originally, the phenomenological concepts of marginal Fermi liquid (MFL) and nested Fermi liquid (NFL) have been introduced in order to explain the deviations in the normal state from the Fermi liquid theory [43,44]. Our results shed more light on this question. In agreement with picture of Ruvalds and co-workers we obtain the $\omega$ - and $T$-dependence of the self-energy mainly due to scattering of the quasiparticles on spin fluctuations which is strongest for a nested Fermi topology. In order to investigate the effect of the self-energy $\Sigma(\mathbf{k}, \omega)$ on the dispersion $\omega_{\mathbf{k}}$ we show in Fig. 20 results for $\operatorname{Im} \Sigma\left(\mathbf{k}_{n}, \omega\right)$ at the wave vector along the nodal line as in the previous paragraph. In the normal state one clearly sees the transition from $\Sigma^{\prime \prime}(\mathbf{k}, \omega) \propto \omega^{2}$ to $\Sigma^{\prime \prime}(\mathbf{k}, \omega) \propto \omega$ for low-lying frequencies for various temperatures. The deviation from Landau's theory results in our picture from strong scattering of the quasiparticles on the spin fluctuations and is expected to disappear at temperatures $T \rightarrow 0$, see inset of Fig. 20. In particular, the changes in the 


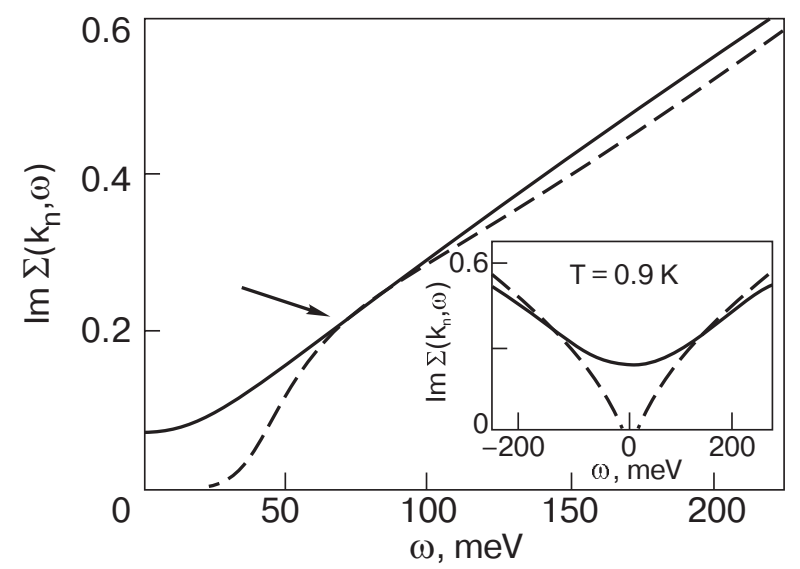

Fig. 20. Calculated frequency dependence of the quasiparticle self-energy at the nodal point $\mathbf{k}_{n}=(0.41 \pi, 0.41 \pi)$ in the first BZ taken from [39] for optimally hole-doped cuprates. The solid curves correspond to the normal state at $T=2 T_{C}$, whereas the dashed curves to the superconducting state at $T=0.5 T_{c}$. In the normal state one clearly sees approximately at $65 \mathrm{meV}$ a crossover from Fermi liquid behavior $\left(\Sigma^{\prime \prime}(\mathbf{k}, \omega) \propto \omega^{2}\right)$ to a non-Fermi-liquid behavior $\left(\Sigma^{\prime \prime}(\mathbf{k}, 0) \propto 0\right)$ for low-energy frequencies as a function of temperature. We show in the inset the behavior of $\Sigma\left(\mathbf{k}_{n}, \omega\right)$ calculated at very low temperatures without superconductivity ( $T \cong 0.9 \mathrm{~K}$, dashed curve). Note, the behavior of the self-energy will become more Fermi liquid like in the overdoped regime and thus a kink feature will be less pronounced.

velocity of quasiparticles are determined in EDC as $u_{F}^{*}=u_{F} /\left(1+d \Sigma_{k}^{\prime} / d \omega\right)$ versus frequency. At frequencies around $65 \mathrm{meV}$, the real part of $\Sigma(\mathbf{k}, \omega)$ shows a flattening as can be seen via a Kramers-Kronig analysis of $\operatorname{Im} \Sigma(\mathbf{k}, \omega)$. Therefore, at this frequency the effect of the scattering on spin fluctuations starts to reduce. Then, a more Fermi-liquid-like behavior can be found.

In Fig. 21 we also demonstrate the feedback of superconductivity on $\Sigma(\mathbf{k}, \omega)$. We expect that it should be strongest for the antinodal direction of the $\mathrm{BZ}$ where the superconducting gap is maximal. Note that the superconducting gap $\Delta(\mathbf{k}, \omega)$ induces changes in the self-energy. Therefore, the superconductivity will induce an extra structure in the kink feature (a so-called s-shape) which should be further verified in the experiment.

To summarize this subsection we have calculated the pronounced momentum and frequency dependence of the quaspiarticle self-energy for various doping concentrations in hole-doped cuprates and find that this results in a kink feature in the renormalized energy dispersion which agrees well with recent ARPES experiments. The origin of this is the coupling of the quasiparticles to spin fluctuations that seems to be the case for cuprates. Another reason for the kink struc-

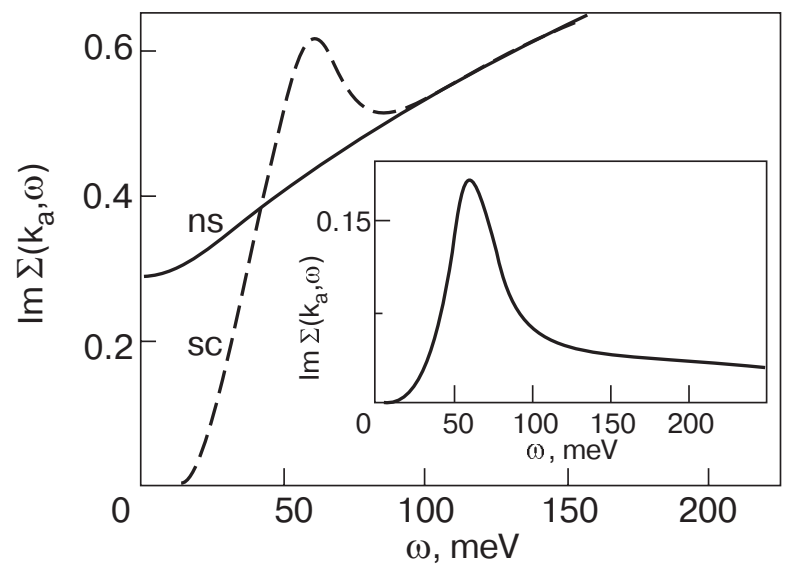

Fig. 21. Calculated frequency dependence of the quasiparticle self-energy at the anti-nodal point $\mathbf{k}_{a}=(0.15 \pi, \pi)$ in the first BZ taken from Ref. 41 for optimally hole-doped cuprates. The solid curve correspond to the normal state at $T=2 T_{C}$, whereas the dashed curves to the superconducting state at $T=0.5 T_{c}$. Inset: the corresponding superconducting gap function $\Delta\left(\mathbf{k}_{a},()\right.$ versus frequency.

ture is a the change in the frequency dependence of the self-energy from non-Fermi-liquid to a Fermi-liquid-like. Due to a different spectrum of the spin fluctuations and fewer density of states the electron-doped cuprates do not exhibits a kink feature.

\section{Conclusions}

In our short review we have covered some important aspects of the doping dependence of many physical properties of cuprates based on a Fermi liquid-like approach. In particular, we have shown that the spinfluctuation mechanism of superconductivity on the basis of a microscopic Eliashberg approach supports the idea that the symmetry of the superconducting order parameter is of $d_{x^{2}-y^{2}}$-wave type. It has been found that the renormalization of the quasiparticle spectrum caused by the scattering on spin fluctuations results in the so-called kink-feature seen in ARPES. Moreover, the spin fluctuations will result in a strong anisotropy of the renormalization at different parts of the $\mathrm{BZ}$ and thus will lead to a strong anisotropy of kink feature which has to be further studied in experimentally and theoretically. Another important result is that the non-Fermi liquid behavior observed in the experiment that led to the suggestion of marginal Fermi-liquid approach can be successfully explained on the basis of a microscopic theory that takes into account the strong scattering of quasiparticles on spin fluctuations.

Another important achievement of the microscopic Eliashberg approach is that the spin excitations spectrum renormalizes below $T_{c}$ due to occurrence of superconductivity with $d$-wave order parameter and 
yields the resonance peak. Furthermore, the topology of the Fermi surface and the momentum dependence of the superconducting gap explains the peculiar dispersion of the resonance peak observed experimentally. The spin fluctuation scenario can also explain many of the properties of cuprates in the underdoped and overdoped cases due to nearness to an antiferromagnetic instability. In the overdoped regime the behavior of cuprates seems to be more Fermi-liquid-like, since the influence of antiferromagnetic fluctuations becomes less important and all features will mainly depend on the superconducting gap. Finally, the most important achievement in recent years was the understanding of the asymmetry of the phase diagram between hole- and electron-doped cuprates. We would like to note, that the results of the FLEX approximation agree to a large extent with the spin-fermion model [45].

On the other hand, one has to mention that the whole problem of high- $T_{c}$ superconductivity is far from being resolved. This concerns, for example, the nature of the pseudogap observed in underdoped cuprates. We did not discuss this problem in this review, since despite of several scenarios which exist at the moment none of them is well accepted. In this connection, one of the main theoretical problems that remains to be understood is the calculation of the dynamical spin susceptibility near half-filling and inclusion of the (localized) short-range antiferromagnetic correlations. Even though, the concept of Cooperpairing due to spin fluctuations seems to be in order for describing physics of cuprates, many further efforts are needed in order to provide a complete picture of high- $T_{C}$ superconductivity and its phase diagram.

We are thankful to M. Sigrist and A. Chubukov for helpful discussions. DM acknowledges the financial support from the Alexander von Humboldt Foundation.

1. D. Scalapino, Phys. Rep. 250, 329 (1995).

2. L.P. Regnault, Ph. Bourges, and P. Burlet, in: Neutron Scattering in Layered Copper-Oxide Superconductors, A. Furrer (ed.), Kluwer Academic Publishers, Dordrecht-Boston-London (1998), p. 85.

3. D. Brinkmann and M. Mali, in: NMR-Basic Principles and Progress, P. Diehl, E. Fluck, H. Gunther, R. Kosfeld, and J. Seelig (eds.), Springer (1994), v. 31, p. 171.

4. J. Hwang, T. Timusk, and G.D. Gu, Nature 427, 714 (2004).

5. T. Timusk and B. Statt, Rep. Prog. Phys. 62, 61 (1999).

6. A.A. Varlamov, G. Balestrino, E. Dilani, and D.V. Livanov, Adv. Phys. 48, 655 (1999).
7. Y. Wang, N.P. Ong, Z.A. Xu, T. Kakeshita, S. Uchida, D.A. Bonn, R. Liang, and W.N. Hardy, Phys. Rev. Lett. 88, 257003 (2002).

8. J.L. Tallon, C. Bernhard, H. Shaked, R.L. Hittermann, and J.D. Jorgensen, Phys. Rev. B51, 12911 (1995).

9. See for review C.C. Tsuei and J.R. Kirtley, Rev. Mod. Phys. 72, 969 (2000).

10. D. Vollhardt and P. Wölfle, The Superfluid Phases of Helium 3, Taylor \& Francis, London (1990).

11. P. Monthoux, A.V. Balatsky, and D. Pines, Phys. Rev. Lett. 67, 3448 (1991).

12. A.J. Millis, H. Monien, and D. Pines, Phys. Rev. B42, 167 (1990).

13. H.F. Fong, P. Bourges, Y. Sidis, L.P. Regnault, J. Bossy, A. Ivanov, D.L. Milius, I. A. Aksay, and B. Keimer, Phys. Rev. B61, 14773 (2000).

14. J. Rossat-Mignod, L.P. Regnault, C. Vettier, P. Bourges, P. Burlet, J. Bossy, Physica C185-189, 86 (1991); H.A. Mook, M. Yethiraj, G. Aeppli, T.E. Mason, and T. Armstrong, Phys. Rev. Lett. 70, 3490 (1993); H.F. Fong, B. Keimer, P.W. Anderson, D. Reznik, F. Dogan, and I.A. Aksay, Phys. Rev. Lett. 75, 316 (1995); ibid. B54, 6708 (1996); P. Bourges, L.P. Regnault, Y. Sidis, and C. Vettier, Phys. Rev. B53, 876 (1996).

15. H. He, Y. Sidis, P. Bourges, G.D. Gu, A. Ivanov, N. Koshizuka, B. Liang, C.T. Lin, L.P. Regnault, E. Schoenherr, and B. Keimer, Phys. Rev. Lett. 86, 1610 (2001).

16. H. He, P. Bourges, Y. Sidis, C. Ulrich, L.P. Regnault, S. Pailhes, N.S. Berzigiarova, N.N. Kolesnikov, and B. Keimer, Science 295, 1045 (2002).

17. S. Pailhes, Y. Sidis, P. Bourges, V. Hinkov, A. Ivanov, C. Ulrich, L.P. Regnault, and B. Keimer, Phys. Rev. Lett. 93, 167001 (2004); D. Reznik, P. Bourges, L. Pintschovius, Y. Endoh, Y. Sidis, T. Masui, and S. Tajima, Phys. Rev. Lett. 93, 207003 (2004).

18. S.M. Hayden, H.A. Mook, P. Dai, T.G. Perring, and F. Dogan, Nature (London) 429, 531 (2004).

19. C. Stock, W.J.L. Buyers, R.A. Cowley, P.S. Clegg, R. Coldea, C.D. Frost, R. Liang, D. Peets, D. Bonn, W.N. Hardy, and R. J. Birgeneau, Phys. Rev. B71, 024522 (2005); C. Stock, W.J.L. Buyers, R. Liang, D. Peets, Z. Tun, D. Bonn, W.N. Hardy, and R.J. Birgeneau, Phys. Rev. B69, 014502 (2004).

20. V. Hinkov, S. Pailhes, P. Bourges, Y. Sidis, A. Ivanov, A. Kulakov, C.T. Lin, D.P. Chen, C. Bernhard, and B. Keimer, Nature 430, 650 (2004).

21. T. Moria and K. Ueda, Adv. Phys. 49, 555 (2000).

22. A. Lanzara et al., Nature (London) 412, 510 (2001).

23. P.D. Johnson, T. Valla, A.V. Fedorov, Z. Yusof, B.O. Wells, Q. Li, A.R. Moodenbaugh, G.D. Gu, N. Koshizuka, C. Kendziora, Sha Jian, and D. G. Hinks, Phys. Rev. Lett. 87, 177007 (2001).

24. A. Kaminski, M. Randeria, J.C. Campuzano, M.R. Norman, H. Fretwell, J. Mesot, T. Sato, T. Takahashi, and K. Kadowaki, Phys. Rev. Lett. 86, 1070 (2001). 
25. S.V. Borisenko, A.A. Kordyuk, T.K. Kim, A. Koitzsch, M. Knupfer, J. Fink, M.S. Golden, M. Eschrig, H. Berger, and R. Follath, Phys. Rev. Lett. 90, 207001 (2003).

26. A.A. Kordyuk et al., Phys. Rev. Lett. 92, 257006 (2004).

27. N.E. Bickers, D.J. Scalapino, and S.R. White, Phys. Rev. Lett. 62, 961 (1989); N.E. Bickers and D. Scalapino, Ann. Phys. 193, 206 (1989); P. Monthoux and D.J. Scalapino, Phys. Rev. Lett. 72, 1874 (1995).

28. T. Dahm and L. Tewordt, Phys. Rev. Lett. 74, 793 (1995);

29. M. Langer, J. Schmalian, S. Grabowski, and K.-H. Bennemann, Phys. Rev. Lett. 75, 4508 (1995).

30. S.T. Lenck, J.P. Carbotte, and R.C. Dynes, Phys. Rev. B50, 10149 (1994).

31. Yu.A. Izyumov, Phys. Usp. 42, 215 (1999).

32. D. Manske, Theory of Unconventional Superconductors - Cooper Pairing Mediated by Spin Excitations, Springer Tracts in Modern Physics, Springer Berlin, Heidelberg (2004), v. 202; D. Manske, I. Eremin, and K.H. Bennemann, in: The Physics of Superconductors, Vol II Superconductivity in Nanostructures, High-T, and Novel Superconductors, Organic Superconductors, K.H. Bennemann and J.B. Ketterson (eds.), Springer-Verlag, Berlin-Heidelberg (2004).

33. D.M. King, Z.-X. Shen, D.S. Dessau, B.O. Wells, W.E. Spicer, A.J. Arko, D. S. Marshall, J. DiCarlo, A.G. Loeser, C.H. Park, E.R. Ratner, J.L. Peng, Z.Y. Li, and R.L. Greene, Phys. Rev. Lett. 70, 3159 (1993).
34. D. Manske, I. Eremin, and K.H. Bennemann, Phys. Rev. B63, 054517 (2001).

35. I. Eremin, D.K. Morr, A.V. Chubukov, K.H. Bennemann, and M.R. Norman, Phys. Rev. Lett. 94, 147001 (2005)

36. T. Ito, K. Takenaka, and S. Uchida, Phys. Rev. Lett. 70, 3995 (1993).

37. I. Eremin and D. Manske, Phys. Rev. Lett. 94, 067006 (2005); A.P. Schnyder, D. Manske, C. Mudry, and M. Sigrist, cond-mat/0510790 (unpublished).

38. J.M. Tranquada, H. Woo, T.G. Perring, H. Goka, G.D. Gu, G. Xu, M. Fujita, and K. Yamada, Nature 429, 534 (2004).

39. D. Manske, I. Eremin, and K.H. Bennemann, Phys. Rev. Lett. 87, 177005 (2001).

40. D. Manske and K.H. Bennemann, Physica C341-348, 285 (2000).

41. A. Kaminski, M. Randeria, J.C. Campuzano, M.R. Norman, H. Fretwell, J. Mesot, T. Sato, T. Takahashi, and K. Kadowaki, Phys. Rev. Lett. 86, 1070 (2001).

42. N.J. Curro, T. Imai, C.P. Slichter, and B. Dabrowski, Phys. Rev. B56, 877 (1997).

43. C.M. Varma et al., Phys. Rev. Lett. 63, 1996 (1989).

44. J. Ruvalds, C.T. Rieck, S. Tewari, J. Thoma, and A. Virosztek, Phys. Rev. B51, 3797 (1995).

45. A.V. Chubukov, D. Pines, and J. Schmalian, in: The Physics of Superconductors, Vol II Superconductivity in Nanostructures, High-T, and Novel Superconductors, Organic Superconductors, K.H. Bennemann and J.B. Ketterson (eds.), Springer-Verlag, Berlin-Heidelberg (2004). 